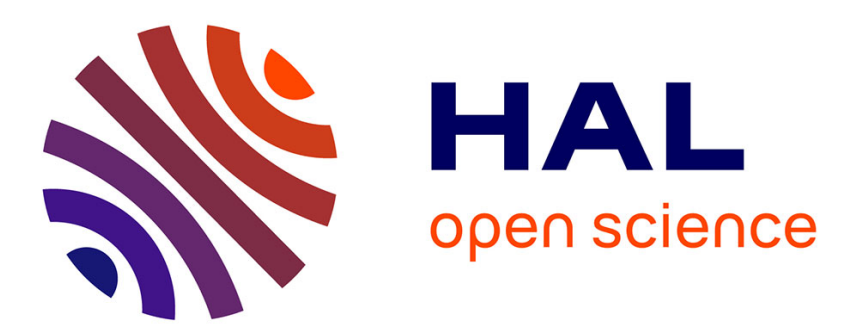

\title{
Understanding and predicting physiological performance of organisms in fluctuating and multifactorial environments
}

Apostolos-Manuel Koussoroplis, Sylvain Pincebourde, Alexander Wacker

\section{- To cite this version:}

Apostolos-Manuel Koussoroplis, Sylvain Pincebourde, Alexander Wacker. Understanding and predicting physiological performance of organisms in fluctuating and multifactorial environments. Ecological monographs, 2017, 87 (2), pp.178-197. 10.1002/ecm.1247 . hal-01693385

\section{HAL Id: hal-01693385 \\ https://hal.science/hal-01693385}

Submitted on 26 Jan 2018

HAL is a multi-disciplinary open access archive for the deposit and dissemination of scientific research documents, whether they are published or not. The documents may come from teaching and research institutions in France or abroad, or from public or private research centers.
L'archive ouverte pluridisciplinaire HAL, est destinée au dépôt et à la diffusion de documents scientifiques de niveau recherche, publiés ou non, émanant des établissements d'enseignement et de recherche français ou étrangers, des laboratoires publics ou privés. 


\title{
CONCEPTS \& SYNTHESIS
}

EMPHASIZING NEW IDEAS TO STIMULATE RESEARCH IN ECOLOGY

\section{Understanding and predicting physiological performance of organisms in fluctuating and multifactorial environments}

\author{
Apostolos-Manuel Koussoroplis, ${ }^{1,3}$ Sylvain Pincebourde, ${ }^{2}$ and Alexander Wacker ${ }^{1}$ \\ ${ }^{1}$ Theoretical Aquatic Ecology \& Ecophysiology, Institute of Biochemistry and Biology, University of Potsdam, \\ Am Neuen Palais 10, Maulbeerallee 2, D-14469, Potsdam, Germany \\ ${ }^{2}$ Faculté des Sciences et Techniques, Institut de Recherche sur la Biologie de l'Insecte (IRBI, CNRS UMR 7261), \\ Université François Rabelais, 37200 Tours, France
}

\begin{abstract}
Understanding how variance in environmental factors affects physiological performance, population growth, and persistence is central in ecology. Despite recent interest in the effects of variance in single biological drivers, such as temperature, we have lacked a comprehensive framework for predicting how the variances and covariances between multiple environmental factors will affect physiological rates. Here, we integrate current theory on variance effects with co-limitation theory into a single unified conceptual framework that has general applicability. We show how the framework can be applied (1) to generate mathematically tractable predictions of the physiological effects of multiple fluctuating co-limiting factors, (2) to understand how each co-limiting factor contributes to these effects, and (3) to detect mechanisms such as acclimation or physiological stress when they are at play. We show that the statistical covariance of co-limiting factors, which has not been considered before, can be a strong driver of physiological performance in various ecological contexts. Our framework can provide powerful insights on how the global change-induced shifts in multiple environmental factors affect the physiological performance of organisms.
\end{abstract}

Key words: co-limitation; covariance; eco-physiology; feeding rate; global change; multiple stressors; nonlinear averaging; nutrients; scale transition; temperature; temporal ecology; variance.

\section{INTRODUCTION}

Variation is the norm in nature. The natural environment is heterogeneous across space, time, and scales. Yet, ecologists mostly focus on the mean environmental conditions as a predictor variable of the patterns and processes they observed. There is, however, an increasing recognition that the descriptors of natural heterogeneity, such as the statistical variance and the probability distribution of environmental conditions, explain many important ecological phenomena (Benedetti-Cecchi et al. 2006). For example, understanding how variance influences organismal physiological performance is essential for predicting the impacts of variable plant phytochemistry on herbivores (Underwood 2004, Hood and Sterner 2010, Wetzel et al. 2016) and increasing temperature variation on ectotherms (Paaijmans et al. 2010, Estay et al. 2014, Vasseur et al. 2014).

Manuscript received 21 July 2016; revised 17 November 2016; accepted 7 December 2016. Corresponding Editor: Aimée T. Classen.

3E-mail: apostolos.koussoroplis@uni-potsdam.de
Environmental variation can be experienced at the population level, i.e., individuals or groups of individuals experience different values of a limiting factor depending on their position in the landscape. However, quite often variation can also be experienced at the individual level, i.e., the same individual faces changing values of a limiting factor within its lifetime. Hence, the effects of the environmental variation (e.g., temperature, light, $\mathrm{pH}$, oxygen, food quantity and quality) on physiological performance should be analyzed at the scales relevant to individual organisms (Potter et al. 2013). This variation can be spatial (e.g., movements within a thermally heterogeneous landscape) and/or temporal (e.g., daily temperature fluctuations). Even very small organisms such as spider mites $(<1 \mathrm{~mm})$ moving across a single leaf can experience microhabitats that differ by more than $10^{\circ} \mathrm{C}$ (Caillon et al. 2014). Similarly, within an hour, understory plants or phytoplankton cells might experience changes in irradiance that span from near darkness to oversaturating light (Ruel and Ayres 1999, Litchman 2000, Retkute et al. 2015). Finally, the food quantity and quality (essential nutrients, secondary metabolites) encountered by consumers during 
their lifetime can also be highly variable (Park et al. 2004, Simpson and Raubenheimer 2012). Even consumers foraging within individual plants experience substantial variation in nutritional quality (Orians and Jones 2001). A growing body of experimental and theoretical work demonstrates that there is much to be gained in the study of the sources and consequences of the variation experienced by individual organisms (Dowd et al. 2015, Pincebourde et al. 2016).

To date however, studies on variation mostly considered single environmental factors, and to a large extent only temperature, thus largely ignoring the fact that organismal performance can be simultaneously colimited by several factors. Here, co-limitation is defined (see the glossary in Box 1 for definitions) in its broadest sense: when the combination of simultaneous or sequential changes in several biotic or abiotic factors has a different effect on organismal performance response (e.g., vital rates such as growth, maturation, fertility, survival) than the effects of changing each factor alone. To cite only a few examples, such co-limitations have been repeatedly shown for light and temperature on plants (Edwards et al. 2016), temperature and nutrients (Cross et al. 2015), or various combinations of nutrients (Harpole et al. 2011, Sperfeld et al. 2016) on autotrophs and heterotrophs. Co-limitation of physiological performance does not necessarily involve two distinct factors (e.g., light and temperature), but can also involve the same factor in different contexts (e.g., temperature during immersion and emersion in intertidal organisms; Pincebourde et al. 2012). Interestingly, co-limitation can involve a fluctuating factor and the temporal scale at which the factor fluctuates as co-limiting factor (Kingsolver and Woods 2016). Despite accumulating evidence on the importance of co-limitation on physiological performance of organisms, a conceptual framework of how organisms can be affected by environmental variation in their colimiting factors is still missing (Gunderson et al. 2016). Yet, such a framework is essential for addressing the pressing issue of the potential effects of the multiple drivers of ecological change on organisms (Darling and Côté 2008, Jackson et al. 2016).

Our objective here is to introduce a conceptual framework in physiological ecology that motivates and guides the study of the role of multifactorial environmental variation. The framework (Fig. 1) extends recent theory on the consequences of the thermal variation experienced by individual organisms (Dowd et al. 2015) to any combination of variable factors that might simultaneously limit performance. In the first part of the paper, we propose a novel integration of theory on nonlinear averaging in biological systems (Chesson 2012, Denny and BenedettiCecchi 2012, Dowd et al. 2015), co-limitation theory concepts (Harpole et al. 2011, Sperfeld et al. 2016), and

\section{Box 1. Glossary.}

Additivity: When the effect of simultaneous or sequential changes in several biotic or abiotic factors equals the sum of the effects of changing each factor alone.

Antagonism: When the effect of simultaneous or sequential changes in several biotic or abiotic factors is smaller than the sum of the effects of changing each factor alone.

Co-limitation: When the simultaneous or sequential changes in several biotic or abiotic factors have a different effect on performance than the effects of changing each factor alone.

Covariance effect: Sensitivity of the performance to the covariance of the co-limiting factors in a variable environment.

Cross-dependence: When the level of one of the co-limiting factors determines the shape (i.e., the position of the maxima, minima, inflexion, and/or half-saturation points along the limiting factor axis) of the response to the other factor.

Effect partitioning (scale transition [ST] theory): Mathematical partition of the ST term that allows the quantification of the individual contributions of the variances of each co-limiting factor as well as that of their covariance.

Integrated performance (ST theory): Predicted performance in a variable environment. Symbolized by $\overline{f(x)}$.

Mean-field (ST theory): Biological response (here, organismal performance) in a constant environment. Symbolized by $f(\bar{x})$.

Scale transition theory: A mathematical upscaling "recipe" stemming from recognition that biological response functions are typically nonlinear and that the interaction of these nonlinearities with spatial or temporal variation distorts the prediction of large-scale patterns from small-scale patterns.

Scale transition term (ST theory): The mathematical correction to add to the mean-field in order to predict the integrated performance.

Static performance curve: The common practice of experimentally generating a curve describing the performance of an organism as a function of a limiting factor under constant conditions. While several factor levels are tested, the level of the factor experienced by individual organisms over the experiment is kept constant.

Synergy: When the effect of simultaneous or sequential changes in several biotic or abiotic factors is larger than the sum of the effects of changing each factor alone. 


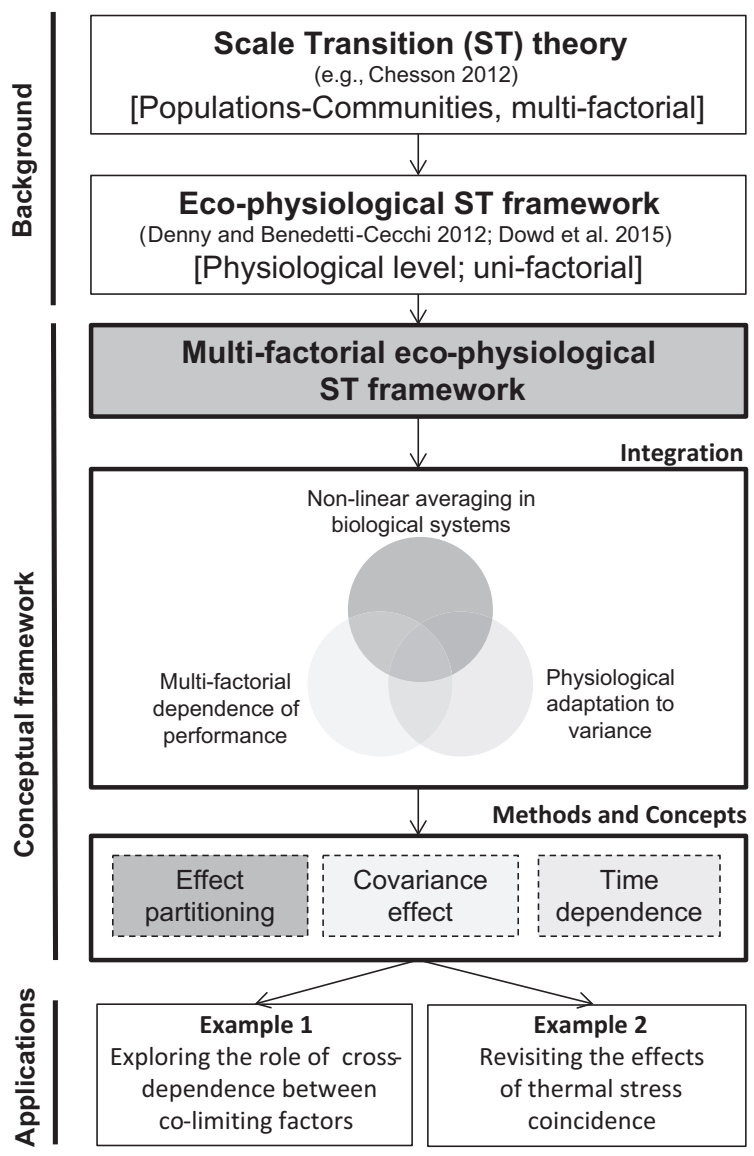

FIG. 1. Conceptual diagram of the structure of this paper. The diagram shows the place of our eco-physiological, multifactorial, scale transition (ST) framework in the literature and the ideas and concepts that it integrates. The main novel methods and concepts that our framework contributes are illustrated through two examples.

phenotypic plasticity of the responses to variance (Kingsolver and Woods 2016, Sinclair et al. 2016). After presenting the mathematical framework for predicting the effect of multifactorial variation and its utility for partitioning the contribution of each factor (effect partitioning; Fig. 1, Box 1), we focus on the contribution of the covariance between factors (covariance effect; Fig. 1), which remains virtually unconsidered in eco-physiology (Pincebourde et al. 2012, Koussoroplis and Wacker 2016). The mathematical framework has to be viewed as a nullmodel based on certain assumptions on the physiology of the organisms, which when violated, lead to deviations from predictions. We review the broad categories of such deviations and provide a novel synthetic view of how these deviations relate to the temporal scale at which variance manifests (time dependence; Fig. 1). We propose this synthesis as a diagnostic tool for identifying the specific physiological mechanisms causing the deviation.

In the second part of the paper, we present two application examples of our conceptual framework. In the first example, we explore a novel question: How does the complexity of the interaction between co-limiting factors (cross-dependence) modulate the way different statistical moments of the experienced environment affect physiological performance? The second example illustrates how the problem of coincidence of environmental stressors can be reformulated within our framework and the novel insights to be gained from that. In both examples, the covariance between co-limiting factors is predicted to be an important driver of physiological performance. We conclude by discussing how our framework can conceptually advance global change research and identify the necessary future theoretical and methodological directions.

\section{THEORY AND CONCEPTS}

\section{Nonlinearity of physiological responses and environmental variance}

One of the main mechanisms through which organismal performance is affected by environmental variation is the nonlinearity that characterizes most of the physiological responses to the various environmental factors (Ruel and Ayres 1999). For example, temperature performance curves (TPC) are generally characterized by an exponential increase at low temperatures, a transition to a peak at an optimal temperature, followed by a rapid decline in performance at higher temperatures. In thermally variable environments, the nonlinearities of the TPC lead to disproportionate effects of cool and warm events on performance. Because of Jensen's inequality (Jensen 1906), the integrated performance over a period of thermal variability increases (concave upward part of the TPC) or decreases performance (concave downward part of the TPC) relative to that predicted by mean temperature (Ruel and Ayres 1999).

The quantification of these increases or decreases can be achieved using mean-field approaches (Morozov and Poggiale 2012), which are the core of the scale transition (ST) theory. This theory focuses on the changes in the equations for population dynamics as the spatial or temporal scale enlarges (Chesson 2012). There is however a formidable opportunity to apply the concepts of this theory to other levels of biological organization such as the physiological level (Denny and Benedetti-Cecchi 2012). Such an "eco-physiological" ST framework has been recently developed (Box 2) for organisms limited by a single factor (temperature), allowing important theoretical insights on the effects of thermal variability on ectotherms (Dowd et al. 2015). However, despite the fact that the vital rates of organisms can be limited by multiple environmental factors simultaneously, current theory does not include co-limitation. Here, we address this theoretical gap.

\section{Accounting for multiple co-limiting factors and partitioning their effects}

A strength of the ST mathematical framework is that it can be extended to include as many co-limiting factors 


\section{Box 2. Using scale transition (ST) theory for predicting physiological performance in fluctuating unifactorial environments. \\ Theoretical principles}

In its ecophysiological version (Dowd et al. 2015), ST theory (Chesson et al. 2005) predicts that the physiological performance in a variable environment is expressed as the sum of the performance under the average yet constant environment (the mean-field) and a correction term (the ST) that accounts for the effect of environmental variance on performance. For a performance function, $g$, (or any other vital rate function contributing to organismal fitness) depending on the abundance of a resource, $R$, the ST can be written as a second-order Taylor expansion around the mean resource value:

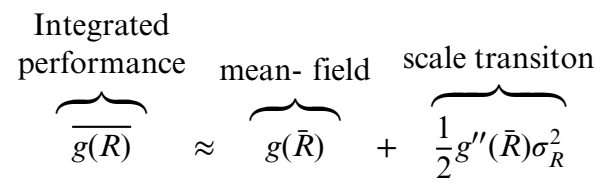

where $\overline{g(R)}$ is the integrated performance under variable $R$ conditions and $g(\bar{R})$ is the growth expected on the averaged resource over the considered time period, $\bar{R} \cdot g^{\prime \prime}(\bar{R})$ is the second derivative of $g$ and quantifies the nonlinearity of the performance function measured at $\bar{R}$. The variance around $\bar{R}$ is $\sigma_{R}^{2}$. Note that $g^{\prime \prime}(\bar{R})>0$ for concave upward functions, and $g^{\prime \prime}(\bar{R})<0$ for concave downward functions, thus explaining the positive or negative effects of environmental variance, respectively. For linear functions, $g^{\prime \prime}(\bar{R})=0$ thus leading to $\overline{g(R)}=g(\bar{R})$ (no variance effect).

\section{Applications and links to ecological concepts}

Scale transition theory is increasingly applied to thermal biology (recently reviewed by Dowd et al. 2015). For example, it can been used to explain (1) why ectotherm's body temperature preferences should lie below the temperatures that optimize physiological performance (Martin and Huey 2008), (2) why ectotherms fitness should be more vulnerable to changes in temperature variance rather than to averaged climate warming (Vasseur et al. 2014), and (3) why temperature variance has to be accounted for when determining the climatic ranges for the development (Blanford et al. 2013) and the transmission (Paaijmans et al. 2010) of pathogens.

Scale transition theory can also be used to translate risk sensitivity of foraging behavior into long-term energetic gain (Smallwood 1996, Matassa and Trussell 2014). In the context of resource-dependent growth, ST theory can be related to resource- and growth-integration, two important concepts of resource-limited growth in variable environments (Litchman 2000, Sterner and Schwalbach 2001, Hood and Sterner 2010). Growth integrators readily respond to resource abundance changes, producing synchrony between growth and resource fluctuations. The resulting long-term growth rate is the mean of the growth expected for each value the resource takes, thus the $\overline{g(R)}$ term of Eq. 1. Alternatively, resource integrators are able to store resources, thus buffering the resource fluctuations and, by consequence growth fluctuations, around their mean values (Fujiwara et al. 2003). Hence, the integrated growth on variable resources departs from the predicted $\overline{g(R)}$ and converges toward the growth predicted by the mean resource level $g(\bar{R})$ depending on the strength of the storage buffering effect (reserve effect).

Scale transition theory can be applied to both spatial and temporal variations. Rather than quantifying the integrated performance through time of an individual in a variable environment, ST can be used to describe the instantaneous performance of a population of individuals distributed in a heterogeneous landscape. The two applications can be combined to estimate the time-integrated physiological performance of the population.

\section{Limitations}

Scale transition theory is a highly valuable theoretical tool for understanding the effects of environmental variation on physiological performance, the mean-variance interaction, and in its multifactorial version (see text: Accounting for multiple co-limitng factors and partitioning their effects), for partitioning variance and covariance effects. However, the ST equation is accurate only when $\sigma_{x}$ is small. For temperature performance curves (TPCs) for instance, it is accurate only when the range of temperature is small compared to the overall breadth of the TPC. For numerically accurate predictions at larger variances, $\overline{g(x)}$ must be calculated using

$$
\overline{g(x)}=\bar{g}\left(\mu_{x}, \sigma_{x}^{2}\right)=\int P\left(x ; \mu_{x}, \sigma_{x}^{2}\right) g(x) d x
$$

where $P\left(x ; \mu_{x}, \sigma_{x}^{2}\right)$ is the probability density function of the distribution of the limiting factor $x$ (Vasseur et al. 2014). The equation can be solved analytically for Gaussian distributions. For more complex distributions, $\overline{g(x)}$ must be calculated from numerical simulation in which $x$ are drawn at random from the distribution. For each drawn $x$, a $g(x)$ is calculated to produce the arithmetic average $\overline{g(x)}$. Also, in its current version, ST theory is based on performance functions that do not account for multiple limiting factors or some physiological phenomena thereby reducing the accuracy of the predictions under some circumstances (see Box 3: Time-scale-dependent effects). 
as necessary. The ST not only allows predictions of the general effect of multivariate environmental heterogeneity but also on the contribution of each factor to that effect (Melbourne and Chesson 2006). Building on the unifactorial example presented in Box 2, and following (Chesson et al. 2005, Morozov and Poggiale 2012), for a performance function, $g$, depending on the abundance of two variable resources (or any other factors), $R_{1}$ and $R_{2}$, the Taylor expansion can be written as

\section{Integrated}

performance mean- field importantly, the magnitude of this covariance effect is modulated by the term $g_{R 1, R 2}^{\prime \prime}\left(\overline{R_{1}}, \overline{R_{2}}\right)$. Physiologically, this term reflects how the two factors together influence performance. As long as the two factors act non-additively on a vital rate then this term differs from zero, and the performance in heterogeneous environments becomes dependent on the co-limiting factor covariance. In the case of antagonistic (sub-additive) co-limitation effects, $g_{R 1, R 2}^{\prime \prime}\left(\overline{R_{1}}, \overline{R_{2}}\right)$ is negative which means that performance where $g_{R 1, R 1}^{\prime \prime}\left(\overline{R_{1}}, \overline{R_{2}}\right)$ and $g_{R 2, R 2}^{\prime \prime}\left(\overline{R_{1}}, \overline{R_{2}}\right)$ are the second partial derivatives with respect to $R_{1}$ and $R_{2}$ evaluated at $\overline{R_{1}}$ and $\overline{R_{2}}$ and quantify the nonlinearities in the $R_{1}$ and $R_{2}$ dimensions. These nonlinearities interact with the variances $\sigma_{R 1}^{2}$ and $\sigma_{R 2}^{2}$, respectively. The crosspartial derivative with respect to $R_{1}$ and $R_{2}, g_{R 1 R 2}^{\prime \prime}\left(\overline{R_{1}}, \overline{R_{2}}\right)$ evaluated at $\overline{R_{1}}$ and $\overline{R_{2}}$, quantifies the nonlinearity arising from the non-additive effect of the two resources on performance. Finally, the term $\sigma_{R 1, R 2}$ is the covariance of the two resources. The equation can be extended to accommodate as many co-limiting factors as necessary (although the equation quickly becomes very lengthy). Note also that Eq. 1 is an approximation that is accurate when the variances are small relative to the width and the breadth of the response surface (see also Box 2).

Beyond the fact that Eq. 1 predicts the effect of multifactorial variation on performance, it also allows the partitioning of the contribution of each factor to this effect. Indeed, the first two terms of the ST expression quantify the effect of the variance of each of the two factors while the third term quantifies the effect of the covariance between the two factors (Fig. 2). This effect partitioning is one of the main strengths of the ST theory (Melbourne and Chesson 2006), because it allows us to (1) understand and predict how the different statistical moments of the environment experienced by the organisms affect their performance and (2) see how the partition of these effects dynamically changes with the mean experienced environment. In general, mathematical partitions have proven to be extremely useful in ecology and evolution (Fox 2016). The two examples we provide illustrate the utility and the new insights that can be gained by applying the ST effect partitioning.

\section{Introducing covariance effects: the roles of synergy, antagonism, and additivity between co-limiting factors}

The third term of the ST in Eq. 1, which quantifies the covariance effect, shows that the performance in a variable environment not only depends on the means and the variances in the different factors, but also on the correlation between these variances in space or time. More scale transition is negatively correlated with covariance. For synergistic (super-additive) co-limitation effects, $g_{R 1, R 2}^{\prime \prime}\left(\overline{R_{1}}, \overline{R_{2}}\right)$ becomes positive and therefore covariance and performance are positively correlated.

Nutritional co-limitation illustrates how the nonadditivity of the two factors interacts with the covariance of these co-limiting factors. Empirical studies demonstrate that temporal variation in the nutritional resource has a negative effect on consumer growth (Hood and Sterner 2010, Tremmel and Müller 2013). This can be explained by the fact that the relationships between growth and the specific nutrients that determine food quality are usually concave downward (i.e., the second derivative or growth, $g^{\prime \prime}(\bar{R})$ is negative, see Box 1). If the relationship is concave downward for both co-limiting nutrients $\left(g_{R 1, R 1}^{\prime \prime}\left(\overline{R_{1}}, \overline{R_{2}}\right)\right.$ and $\left.g_{R 2, R 2}^{\prime \prime}\left(\overline{R_{1}}, \overline{R_{2}}\right)<0\right)$, then nutritional variance in any or both of the nutrients decreases consumer performance, as observed in studies involving single nutrient limitation. More interestingly however, Eq. 1 also predicts that spatial or temporal covariance of substitutable and essential co-limiting resources (sensu Sperfeld et al. 2016) should influence consumer integrated performance in different ways depending on the overall nutritional balance (Fig. 3).

For example, terrestrial consumers need often to temporally mix prey with strongly imbalanced nutrient compositions (Simpson and Raubenheimer 2012) thereby experiencing negative temporal covariance in the nutrients that are co-limiting their growth (Fig. 3a, b). In such cases the negative effects of nutritional variability should be less important when the nutrients are substitutable (Fig. 3d) than when they are interactively essential (Fig. 3e). Indeed, substitutable nutrients act subadditively on consumer performance $\left(g_{R 1 R 2}^{\prime \prime}\left(\overline{R_{1}}, \overline{R_{2}}\right)<0\right)$ so that when covariance is negative, the product $g_{R 1, R 2}^{\prime \prime}\left(\overline{R_{1}}, \overline{R_{2}}\right) \sigma_{R 1, R 2}$ becomes positive thereby mitigating the negative effect of the first two ST terms of Eq. 2.

Consumers might also be forced to temporally alternate between low and high quality prey in which the two co-limiting nutrients are either simultaneously absent or present, respectively, thereby experiencing positive nutritional covariance (Fig. 3a). Such a situation could occur in vertically migrating zooplankton feeding in different 
a) Homogeneous Heterogeneous

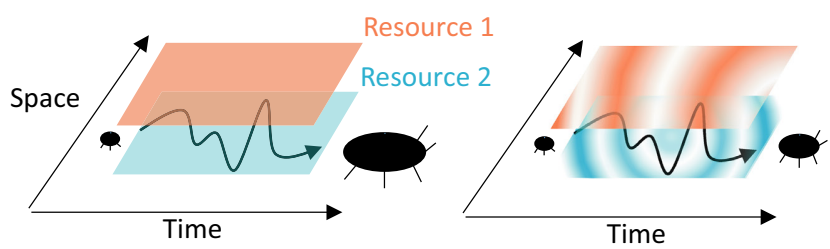

b)

Integrated

$\overbrace{\overline{g\left(R_{1}, R_{2}\right)}}^{\text {performance }} \approx \overbrace{g\left(\overline{R_{1}}, \overline{R_{2}}\right)}^{\text {mean-field }}+\overbrace{\frac{1}{2}[\underbrace{g_{R 1, R 1}^{\prime \prime}\left(\overline{R_{1}}, \overline{R_{2}}\right) \sigma_{R 1}^{2}}_{R 1 \text { variance effect }}+\underbrace{g_{R 2, R 2}^{\prime \prime}\left(\overline{R_{1}}, \overline{R_{2}}\right) \sigma_{R 2}^{2}}_{R 2 \text { variance effect }}+\underbrace{2 g^{\prime \prime}{ }_{R 1, R 2}\left(\overline{R_{1}}, \overline{R_{2}}\right) \sigma_{R 1, R 2}}_{\text {covariance effect }}]}^{\text {Net effect }}$

c) Partitioning heterogeneity effects

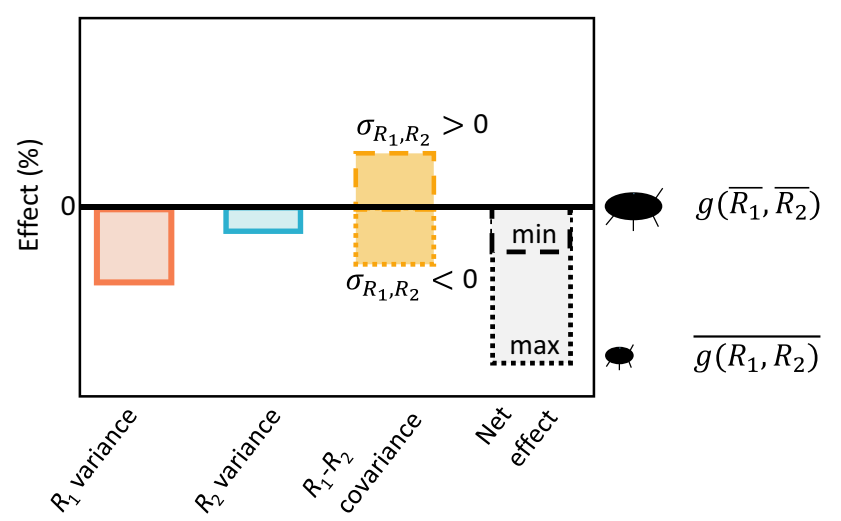

FIG. 2. Partitioning variance effects. (a) The effect of a spatiotemporally heterogeneous environment with two co-limiting factors (resources 1 and 2) on the performance (e.g., growth rate) of an organism is evaluated relative to its performance in a constant environment with the same mean properties (i.e., the mean-field). (b) Using the scale transition equation, the net effect of environmental variance on growth rate can be predicted and partitioned into its components, i.e., the effect of variance of each resource as well as the effect of their covariance. (c) The different effects are expressed as percent deviation from the mean-field. The magnitude and the direction of the covariance effects (and by consequence the net effect of environmental variance) depend on the absolute value and the sign of covariance, respectively.

depths in which either eukaryotic algae or cyanobacteria dominate, the latter being devoid in polyunsaturated fatty acids and sterols, two nutrients known to co-limit zooplankton performance (Sperfeld et al. 2012). Under this scenario, the negative effect of nutritional variability should be less important in the case of strongly limiting essential nutrients (Fig. 3e) because they act superadditively on performance $\left(g_{R 1, R 2}^{\prime \prime}\left(\overline{R_{1}}, \overline{R_{2}}\right) \sigma_{R 1, R 2}\right.$ is positive when covariance is positive).

Finally, generalist consumers typically feeding on a diverse set of prey among which the balance in the two co-limiting nutrients is randomly distributed, should experience a null nutrient covariance. In the absence of a covariance effect the negative effects of nutritional variance are the sum of the $R_{1}$ and the $R_{2}$ variance effects.
Note that co-limiting nutrients do not necessarilly influence performance in the same way all along the twodimensional nutrient space (Fig. 3c) so that the direction of the covariance effect might change. In our example, if the mean level of the two co-limiting essential nutrients is high (Fig. 3c, f), their effect on performance becomes additive $\left(g_{R 1, R 2}^{\prime \prime}\left(\overline{R_{1}}, \overline{R_{2}}\right)=0\right)$ yealding a null covariance effect.

\section{Time-scale-dependent deviations from ST predictions: identifying the underlying mechanisms}

In the simple case when variation is only experienced at the population level (i.e., between individuals), the mathematical framework presented above is sufficient 

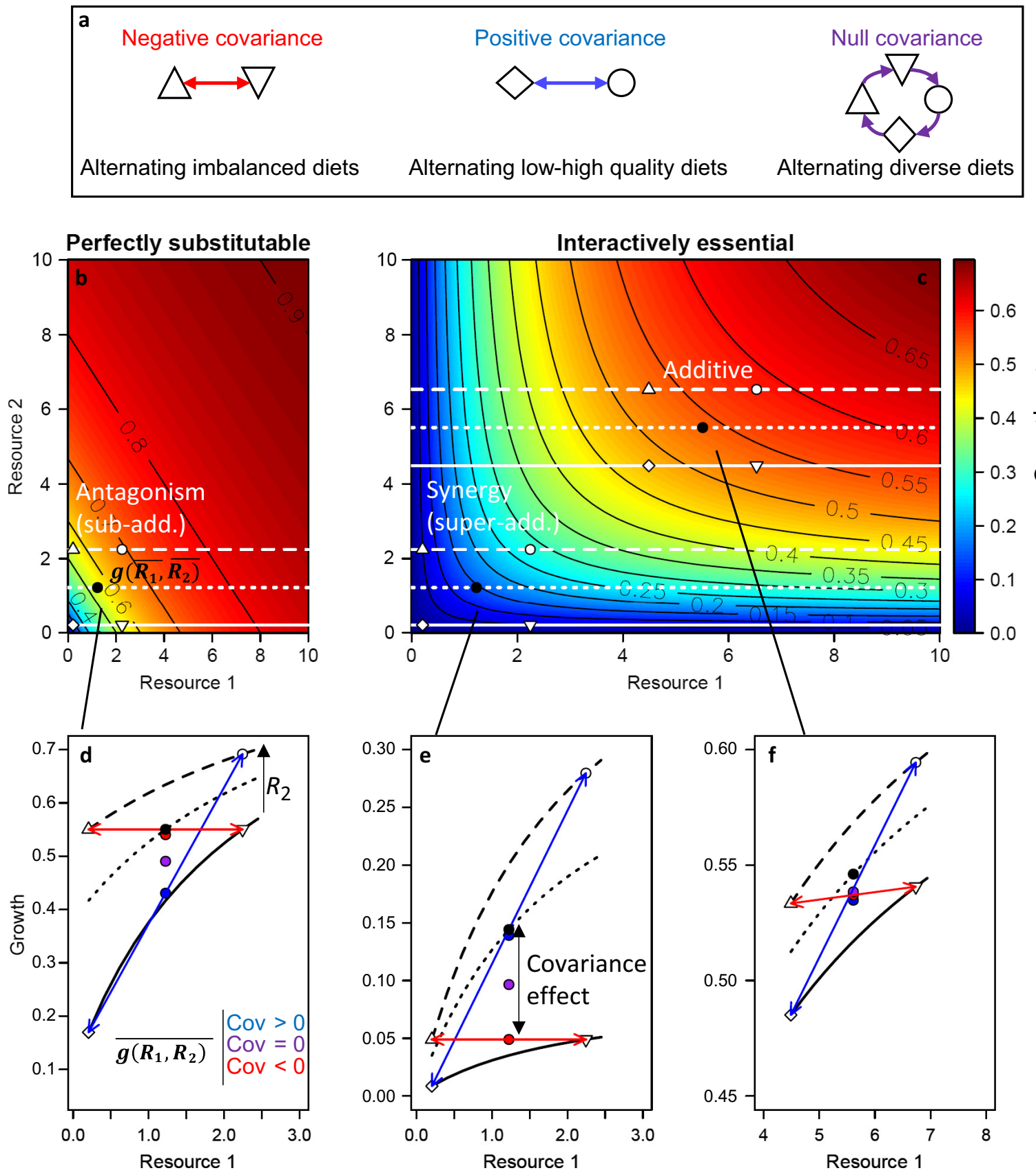

FIG. 3. Covariance effects and the role of synergy, antagonism, and additivity. (a) Depending on the ecological context, consumers may have to deal with different dietary covariance patterns. The different symbols indicate distinct prey composing the diet of a consumer. (b, c) The assignment of the various dietary items on a two-dimensional resource space illustrates how their composition, in terms of co-limiting resource availability differs (arbitrary units). The underlying contour plot depicts the achieved growth rate for constant combinations of the resources, assuming either perfectly substitutable or interactively essential resources (see Sperfeld et al. [2016] for an extensive list of possible co-limitation models). The solid black circles indicate the growth rate ( $\mathrm{d}^{-1}$ ) expected under the average resource availability in the diet when the various items are consumed in equal proportions (mean-field). The effect (sub-additive, super-additive, or additive) of the resources in the considered region of the resource space is written in white. (d-f) Cross-sections of the response surfaces along the white lines in panels b and c (long-dashed, short-dashed, and solid lines, respectively). The different dietary covariance setups are illustrated by the symbols and the double-headed arrows. The predicted integrated performance under the various covariance setups are depicted by the color-filled circles. See Appendix S1 for model equations and parameters. 
for predicting the integrated performance of the average individual. However, when the variation is mainly experienced at the individual level (i.e., across the lifetime of an individual), one needs to additionally account for the effects of the temporal patterns (fluctuation frequency or order of events) of the limiting factor variance (Schulte et al. 2011, Niehaus et al. 2012, Kingsolver et al. 2015). These effects (presented in more detail in Box 3) have been coined as time-dependent effects (Kingsolver et al. 2015) and are the consequences of various physiological functions that evolved to buffer environmental variation (e.g., reserves) or to adjust performance in a variable environment (e.g., phenotypic plasticity/acclimation). Under certain circumstances, time-dependent effects lead to substantial deviations between experimental observations of performance under variable performance and ST predictions. This is because the ST approach is based on performance responses (curves or surfaces) measured under constant conditions (hereafter static performance curves) and assumes that these responses also hold under variable conditions (Litchman 2000, Niehaus et al. 2012, Kingsolver et al. 2015, Sinclair et al. 2016). The deviations might follow the same or the opposite direction than predicted by ST theory, hence amplifying (Pincebourde et al. 2012) or buffering (Kingsolver et al. 2015), respectively, the effects of environmental variation predicted by static performance curves (Box 3).

Some categories of time-dependent effects occur only when the fluctuation frequency is low (acclimation effect; Box 3; Fig. 4c), when the fluctuating factor reaches extreme values (stress effect; Box 3; Fig. 4b), or when the organism deviates dramatically from its growth trajectory (compensation effect; Box 3, Fig. 4d). In other cases, however (reserve and inertia effects; Box 3; Fig. 4a), the effects occur in each situation. Each type of time-dependent effect should cause a predictable deviation pattern from ST predictions (Box 3; Fig. 4a-d). In most cases, the magnitude of this deviation is a function of environmental fluctuation frequency (Box 3; Fig. 4e) so that changing the frequency fluctuation value affects performance, exactly as the focal fluctuating limiting factor does.

The physiological mechanisms underlying timedependent effects operate only within a relatively narrow range of environmental fluctuation frequencies (Box 3; Fig. 4e). If the strongest fluctuations occur outside this frequency range, ST theory can be used to accurately predict organismal performance in variable environments. By contrast, if the limiting factor mostly fluctuates within this range, then ST theory based on static performance curves will have a limited predictive power but can still be useful in another sense. Indeed, the predictions can be regarded as null models to which the realized (observed) performance of individuals and populations need to be compared (Estay et al. 2014). Subsequently, the observed deviations can be used as a diagnostic tool for identifying the specific physiological mechanisms causing these deviations and to reveal when and how they act.
The time-dependent effects described above (Box 3) are found both in univariate and multivariate environments. In the latter, however, the co-limiting factors might interactively influence the rates and the magnitudes of the physiological responses that generate time-dependent effects. For example, the nutritional status of an organism (influenced by the mean and variance in resources) could alter the sensitivity to stressful temperatures (Terblanche et al. 2011) and therefore the onset and the magnitude of a temperature-driven stress effect. Similarly, the dynamics of nutrient reserve accumulation and utilization could have different thermal sensitivities so that the magnitude of the reserve effect depends on whether temperature and food availability covary or not (Koussoroplis and Wacker 2016). In some cases organisms might respond to different stressors with the same physiological mechanisms (Gunderson et al. 2016) generating a very large diversity of possible response patterns. Clearly beyond the scope of the present paper, the possible patterns are discussed elsewhere (Gunderson et al. 2016, Taff and Vitousek 2016).

In conclusion, discarding static performance curves from future research because of time-dependent effects would be counter-productive (Sinclair et al. 2016). These curves are readily available in the literature and are relatively easy to obtain experimentally. Yet, understanding when and how specific time-dependent effects can be expected (Box 3) is essential for making the best out of static performance curves. Based on such knowledge the ST approach can be optimally used, either as a tool for diagnosis or as a tool for prediction.

\section{Application 1: Cross-Dependence Between Co-limiting Factors and Responses to ENVIRONMENTAL VARIATION}

A co-limitation function may differ in whether the two co-limiting factors cross-depend or not. Here, cross-dependence is defined as the influence of the level of a co-limiting factor on the shape of the response to another factor (e.g., inflexion or half-saturation points). In some studies, cross-dependence is termed an "interaction" between the factors (Cross et al. 2015), while in others, "interaction" is used to indicate non-additivity (Sperfeld et al. 2016). We therefore use the term cross-dependence to avoid any confusion. Note however, that co-limitation is also possible without cross-dependence between factors. In this case, the level of one of the colimiting factors influences only the maximum response to the other factor. Whether we have cross-dependence or not is determined by the underlying physiological mechanisms that drive the co-limitation. How crossdependence (or its absence) mediates organismal responses to environmental variation is unknown. Here, we combine effect partitioning with numerical simulations to explore this question within the context of global change biology. 


\section{Box 3. Time-scale-dependent effects.}

\section{Reserve and inertia}

Storage is an ubiquitous feature of living organisms allowing them to store energy, water, or specific nutrients for future use, thus partially decoupling resource fluctuations in the environment from growth (Sterner and Schwalbach 2001, Hood and Sterner 2010). The assimilated energy and nutrients are first transformed into a metabolically inactive pool called reserves and subsequently mobilized to maintenance, growth, and reproduction (Kooijman 2010). Hence, reserves act as a low-pass filter that reduces the amplitude to some extent, or completely mutes high-frequency environmental variation (Muller and Nisbet 2000, Fujiwara et al. 2003). Note that the thermal inertia of the body acts exactly like the reserve effect, decoupling to some extent body temperature from environmental temperature fluctuations (Helmuth et al. 2010).

For concave downward performance responses, reserve should cause observations to deviate from the ST prediction and converge toward the mean-field, thereby mitigating the negative effects of resource variance on physiological performance (Fig. 4a). However, the magnitude of this mitigating effect decreases with decreasing reserve capacity per unit biomass (or decreasing body size for thermal inertia) and with decreasing frequencies of resource (or temperature) fluctuations (Stevenson 1985, Fujiwara et al. 2003). Therefore, the observed performance should tend toward the ST predictions with decreasing fluctuation frequencies (Fig. 4e).

\section{Stress}

The duration of exposure to temperatures (or other factors) that lie far from the thermal optimum may decrease performance with increasing duration of exposure, an effect referred to as thermal stress (Niehaus et al. 2012, Rezende et al. 2014, Kingsolver et al. 2015). The stress is due to various physiological mechanisms (e.g., expression of heat shock proteins) aiming to improve short-term survival but negatively affect long-term performance (e.g., growth, development, or reproduction).

Typically, the static performance curves of organisms are measured over longer temporal scales (several days or weeks, e.g., from birth to sexual maturity) than those at which the organism experiences variability (daily, hourly). Hence, while the static performance curve reflects the potential stress effects, this stress might not occur under fluctuating conditions when the organism is only shortly exposed to the stressful levels of the limiting factor. Consequently, ST predictions overestimate the effect of environmental variance on organismal performance (Fig. 4b). However, the duration of continuous exposure to the stressful factor levels increases when the environmental fluctuation frequency decreases, thereby inducing stress responses. Hence, for decreasing frequencies, observations should tend toward and eventually match the ST predictions (Fig. 4e).

\section{Phenotypic plasticity effects: acclimation and compensation}

When given enough time, organisms might dynamically acclimate their physiology to match the prevailing environmental conditions in order to optimize performance (Taff and Vitousek 2016). As for stress effects, acclimation responses might be reflected in the static performance curves, but may not occur under variable conditions when the environment changes faster than organisms can acclimate. Hence, the organism spends a large amount of time suboptimally acclimated, during which it underperforms. In this case, the ST predictions based on static performance curves will underestimate the effect of environmental variance on organismal performance (Fig. 4c). However, for decreasing frequencies, the organism has increasingly more time to fully acclimate and perform optimally thus causing the observations to tend toward, and eventually match the ST predictions (Fig. 4e)

Phenotypic plasticity in individual performance may also occur under the form of compensatory growth. The general pattern is for individuals that experienced a period of low growth (e.g., due to low food availability or temperature) to enter a phase of growth acceleration when conditions improve (Metcalfe and Monaghan 2001). This is achieved by various mechanisms such as increased resource uptake rates, prolongation of the daily feeding activity or decreasing competitive interactions between individuals (Arendt 1997, Metcalfe and Monaghan 2001, Gurney et al. 2003). Static performance curves do not incorporate compensatory growth effects because the organisms never experience changes in growth conditions. Yet, compensation may occur under variable conditions, leading to higher growth than predicted (Fig. 4d).

Whether compensatory growth responds to environmental fluctuation frequency depends on how the organism "measures" its developmental delay. When it is measured as the time spent growing suboptimally, compensation effects should appear at low fluctuation frequencies causing observations to deviate from predictions and potentially surpassing the mean-field (Fig. 4e). However, the delay might also be assessed as the developmental state reached at a fixed point in time (e.g., determined by seasonal daylight or temperature changes). In this case, compensation responses should be independent from environmental fluctuation frequencies. 

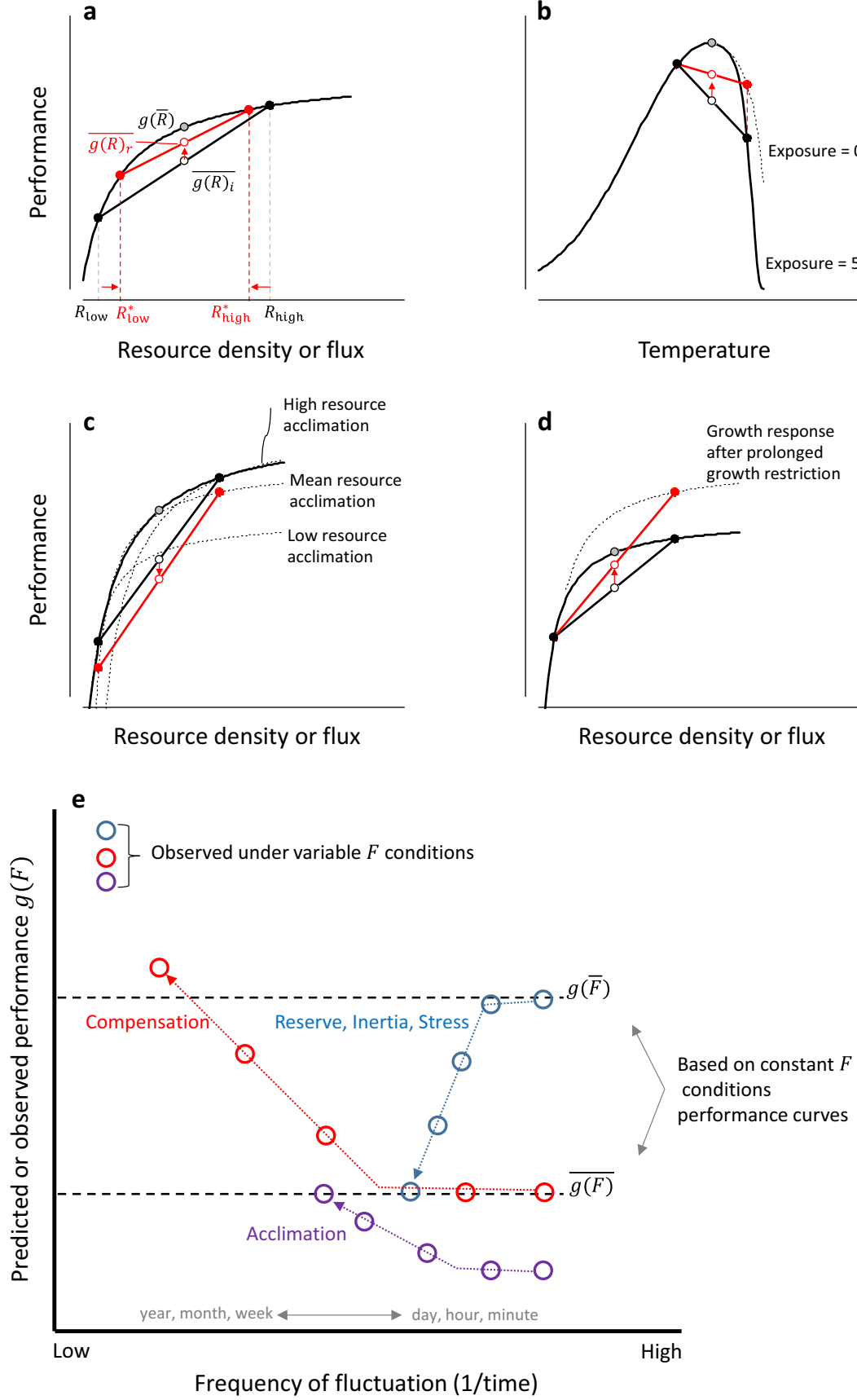

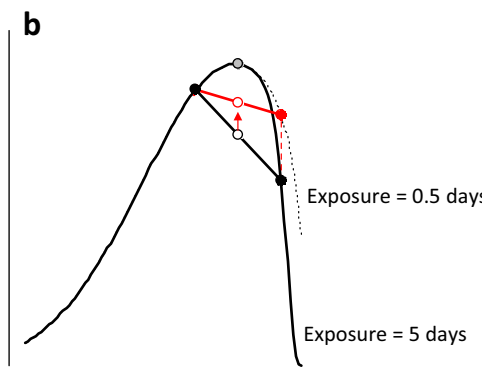

Temperature

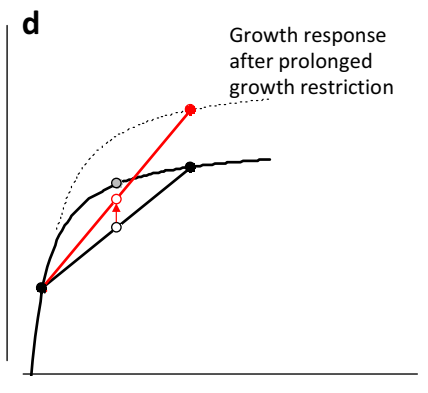

Resource density or flux

FIG. 4. Time-scale effects (see also Box 2). (a) Reserve effect. Environmental resource variance $\left(R_{\text {low }}-R_{\text {high }}\right)$ and resource reserve variance $\left(R_{\text {low }}^{*}-R_{\text {tigh }}^{*}\right)$. (b) Stress effect. The performance depends on exposure duration. (c) Acclimation effect. The organism acclimates to optimize its performance for a given level of the limiting factor (dotted curves). (d) Compensatory growth effect. Following a prolonged period of growth restriction, the organism grows faster than expected (dotted line) under favorable conditions. (e) Influence of fluctuation frequency of the limiting factor $(F)$ on the magnitude of the time-dependent effects. Grayfilled circles are mean-field estimates of performance $g(\bar{R})$; open black circles show inferred performance from static response curves (solid black curves), $g(R)_{i}$. Open red circles are the realized performance, $g(R)_{r}$, under the influence of a time-dependent effect. The different effects do not necessarily manifest within the same range of frequencies.

\section{Temperature-nutrition interactions and global change}

Temperature and nutrient availability are considered key drivers of the rates and the pathways of material and energy movement through ecosystems (Cross et al. 2015). Temperature and nutrition are tightly linked at the physiological level and often co-limit ectotherm performance. Temperature alters the rates of food assimilation and 
energy expenditure thus changing the quantitative needs for food (Lemoine and Burkepile 2012). Temperature also influences the physiological needs of ectotherms for some specific nutrients (Sperfeld and Wacker 2012, Malzahn et al. 2016). Changes in temperature and in resource quantity and quality are expected to occur concomitantly as a result of global changes (Bauerfeind and Fischer 2013, De Senerpont Domis et al. 2014) thus stressing the need to increase our understanding of how these two factors act on ectotherm fitness in a variable world. There are two frequently assumed models for the co-limitation of ectotherm performance by temperature and a resource (sensu Tilman 1982) that could be either energy (food quantity, light) or a specific nutrient (food quality).

\section{Constructing a mean-field model: the multiplicative model}

The first model argues that temperature sets the maximum growth rate of an organism, while the limiting resource levels mediate the realized growth rate. In this model, the maximal possible performance drops with decreasing food availability or quality, yet the shape of the TPC (the position of temperature optimum, critical temperatures, and inflexion points along the temperature axis) remains unchanged across resource abundances (Fig. 5a, solid lines). Furthermore, the model implies that the effect of nutrition is maximal at the optimal temperature, while nutrition has almost no effects close to the extreme temperatures (Fig. 5a, solid lines). This model describes a simple (here multiplicative) co-limitation function where the two factors do not cross-depend. It can be written as

$$
g(T, R)=f(T) \frac{R}{k+R}
$$

where $g(T, R)$ is the realized growth rate (or some other performance parameter), $f(T)$ is the function describing the temperature dependence of growth, $R$ is the availability of the limiting resource, and $k$ a half-saturation coefficient. Note that we model resource dependence of growth as a Monod function, usually applied to nutritional limitation. Light limitation is modelled differently, yet the shape of the response is qualitatively similar to the Monod model as long as no photo-inhibition occurs. Regarding the temperature function, $f(T)$ (see Appendix S2), many alternative formulations are possible, most of them describing a unimodal right-skewed curve (Angilletta 2006).

\section{Constructing a mean-field model: the cross-dependent model}

The second model states that, in addition to setting the maximum growth rate, temperature also affects the nutritional dependence of growth, i.e., how efficiently the organism captures and utilizes resources. In this model, the shape of the TPC changes depending on resource abundance (Fig. 5b, solid lines). This is what we define as a cross-dependent co-limitation and can be written as

$$
g(T, R)=f(T) \frac{R}{k(T)+R}
$$

where $k(T)$ is some function (see Appendix S2) of temperature: linear, accelerating (Rhee and Gotham 1981), or unimodal (Edwards et al. 2016). Applying the ST theory on the two models above allows us to predict the net effect of combined temperature and resource variance around various mean values of the two factors (Fig. 5a, b, dashed lines). Below, we show how the two types of models differ in terms of the mechanisms that drive the effect of environmental variation on performance (Fig. 5c-f).

\section{Partitioning the effects of environmental variation: multiplicative vs. cross-dependent model}

Partitioning the variance effects of the simple colimitation model (Eq. 2) shows that the relative temperature variance effect remains unchanged between resource conditions (Fig. 5c vs. Fig. 5d; Appendix S2: Fig. S3). Similarly, the resource variance effect remains constant across mean temperature conditions (Fig. 5c; Appendix S2: Fig. S3). The implications can be better understood in a hypothetical situation where the resource for consumers within a given habitat is nearly constant but is highly variable among habitats. If the simple colimitation model applies, the nutritional context of the habitat (level of resource limitation) of the consumer should not affect its relative sensitivity to thermal variance (i.e., percent change in performance relative to the constant conditions), which will then solely depend on the mean temperature of the habitat. Mathematically, this can be explained by the fact that the value of the temperature variance effect $\left(\frac{1}{2} g_{T, T}^{\prime \prime}(\bar{T}, \bar{R}) \sigma_{T}^{2}\right.$, Appendix $\mathrm{S} 2$ : Fig. S2) changes at same rate as the mean-field $(g(\bar{T}, \bar{R})$, Appendix S2: Fig. S1) does along a gradient of mean resource. Similarly, the temperature might be constant within habitats but the organism might face important temporal nutritional variance. For the same average level of resource, the relative sensitivity of performance to nutritional variance should remain unaffected across different thermal habitats (Appendix 2: Fig. S3).

In the case of a cross-dependent co-limitation (Eq. 3), the temperature variance effect (Appendix S2: Fig. S2) along a gradient of mean resource changes at a different rate than the mean-field does (Appendix S2: Fig. S1). This implies that the sensitivity to temperature variance changes across resource contexts (Fig. 5e vs. Fig. 5f; Appendix S2: Figs. S3 and S4). In the specific example shown here, the performance of a light or nutritionally limited organism experiencing mean temperatures close to its optimum will be more sensitive to thermal variance than under moderate or no resource limitation. For the same reasons, the negative effect of light or nutritional variance will peak when the mean environmental temperature conditions are around the thermal optimum of the organism (Fig. 5e, f). 
a) Simple (multiplicative) co-limitation

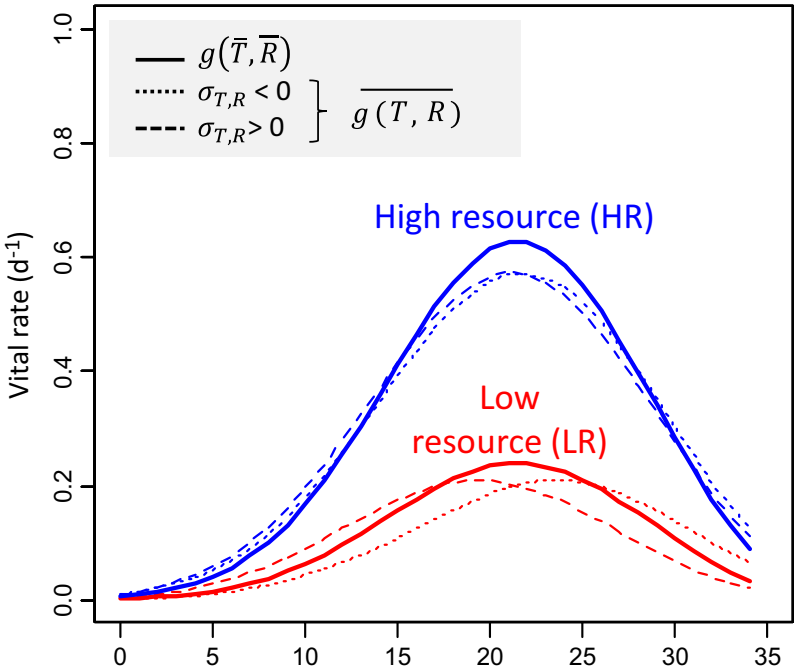

c) LR

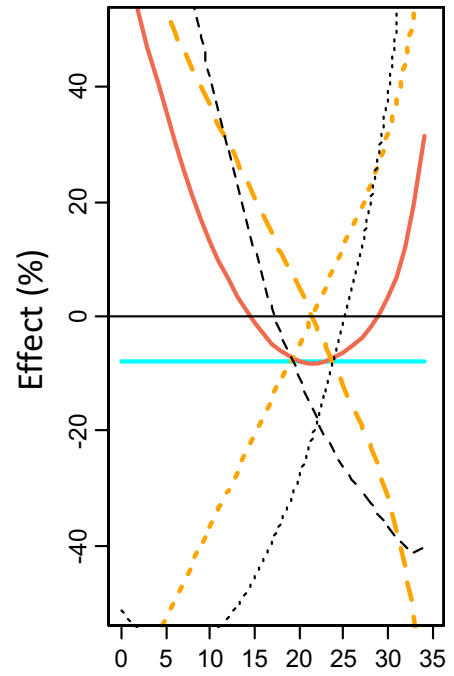

d) $\mathrm{HR}$

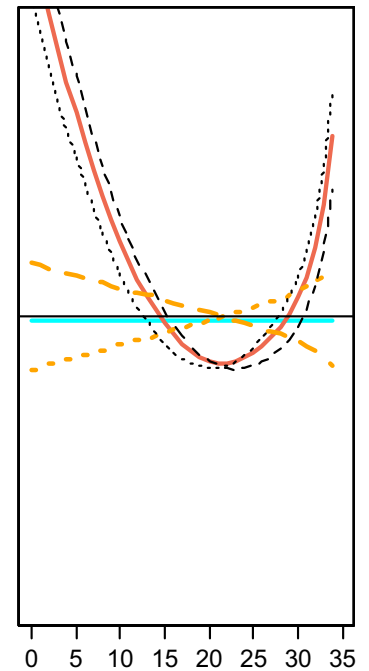

b) Cross-dependent co-limitation

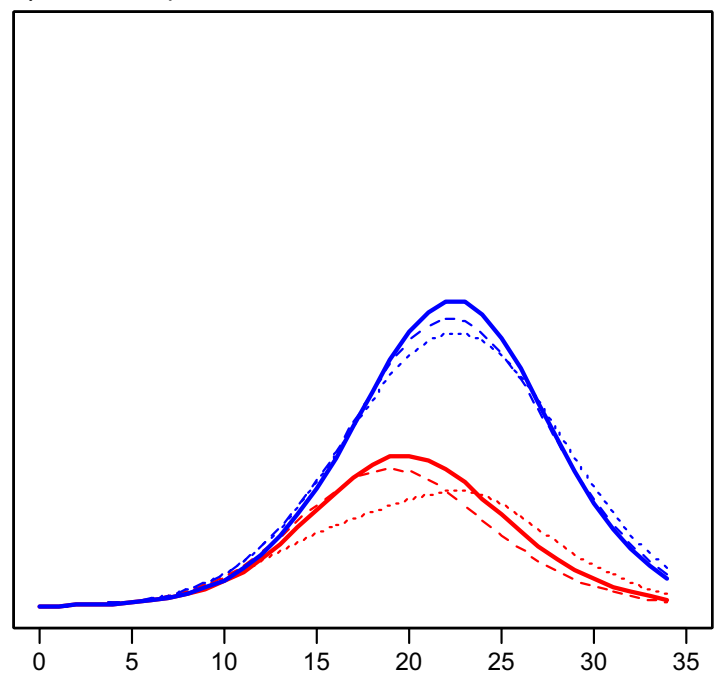

e) LR

f) $\mathrm{HR}$
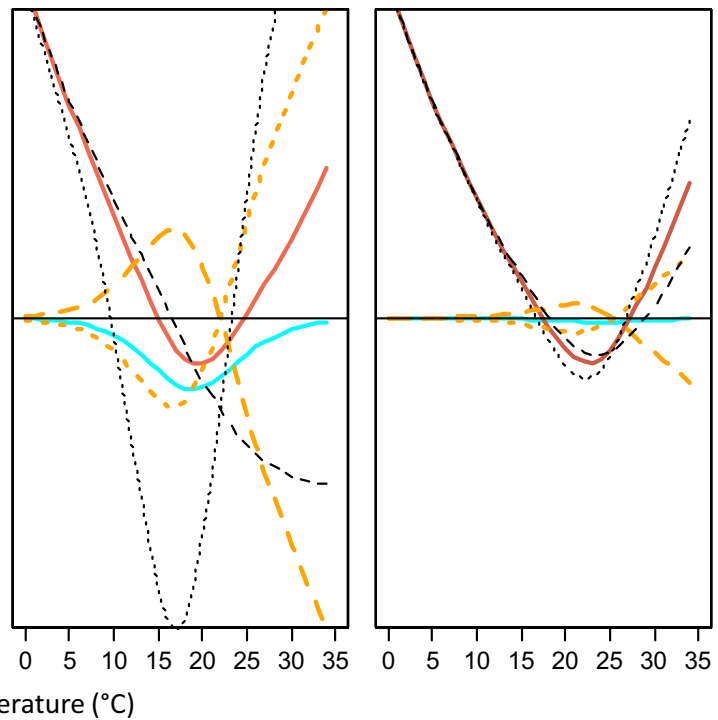

Compound effects

Temperature variance

Resource variance

$\sigma_{T, R}<0$

$\sigma_{T, R}>0$

Covariance
Net effect

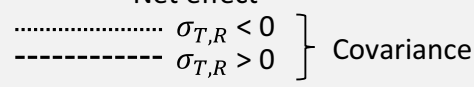

FIG. 5. Exploring the role of cross-dependence between co-limiting factors. Each component effect is derived from Eq. 2. The net effect is the sum of all compound effects. $(\mathrm{a}, \mathrm{b})$ Net effect of joint temperature and resource variance $\left(\sigma_{T}^{2}=4\right.$ and $\sigma_{R}^{2}=0.5$, respectively) on ectotherm performance for the (a) simple and the (b) cross-dependent co-limitation models (Eqs. 2 and 3 , respectively) with severe resource limitation (LR, low resource, $\bar{R}=1$ ) and near saturating resource (HR, high resource, $\bar{R}=4.4$ ). $(\mathrm{c}-\mathrm{f})$ Variance effect partitioning based on Eq. 2 for the simple (c, d) and the cross-dependent (e, f) interaction models. Red, temperature variance effect; blue, resource variance effect; orange, temperature and resource covariance effect; black, net variation effect. The effects are calculated for the maximum and minimum possible covariance (long dashed lines, $\sigma_{T, R}=1.41$, and short dashed lines, $\sigma_{T, R}=-1.41$, respectively). See Appendix S2 for model equations and parameters. 
When both temperature and resource vary, the organism experiences the effects of their covariance (Appendix S2: Fig. S2). Regardless of the co-limitation type (simple or cross-dependent), the sensitivity of performance to covariance changes both along mean temperature and resource gradient. In general, the sensitivity to covariance increases as the resource becomes more limiting (Fig. 5c, f; Appendix S2: Fig. S3). Yet, the patterns along the temperature gradient strongly differ between the two co-limitation models. In the simple co-limitation, the sensitivity to covariance increases monotonically as the temperature moves away from the thermal optimum and toward the lower boundary of the TPC. In the case of cross-dependent co-limitation, the sensitivity to covariance peaks near the thermal optimum. It becomes null at the optimum and then above the optimum increases again but with an inversed direction.

In conclusion, the co-limitation model clearly determines how the effects of each co-limiting factor mix in the various regions of the state-space to determine the net effect on physiological performance. Hence, knowledge of the underlying co-limitation model is essential for determining the mechanism through which changes in environmental heterogeneity alter organismal performance.

\section{Application 2: Revisiting the Effects of Thermal Stress Coincidence}

Here, we present a second example of how our framework can be used to infer the mechanisms at play during the response of organisms to multifactorial and variable environments using a published experimental study (Pincebourde et al. 2012). In this study, the authors were interested in the effects of the degree of coincidence (within a tidal cycle) of extreme, yet non-lethal aerial and underwater body temperatures (emersed $\left[T_{\mathrm{e}}\right]$ and immersed $\left[T_{\mathrm{i}}\right]$, respectively) on the long-term feeding rate of an intertidal predator (Pisaster ochraceus). This study controlled the mean and variance effects to show that the temporal pattern of emersed temperatures relative to immersed temperatures influenced predation rate rather counter-intuitively.

First, we show how the experimental design of the study can be translated in the mathematical terms used in the present framework and generate mechanistically justified null hypotheses. Second, we use the effect partitioning to gain novel theoretical insight on how the effects of thermal stress coincidence depend on mean $T_{\mathrm{i}}$ and $T_{\mathrm{e}}$ conditions. Finally, we use the conceptualization of the time-dependent effects presented above to provide a more solid interpretation of the deviations of the experimental observations from the null hypotheses, which was not possible to infer outside the proposed framework.

\section{Constructing a mean-field model}

The first step for applying ST theory is to describe the performance as a function of the focal factors in a mathematical model. Following the logic in Pincebourde et al. (2012), we treated $T_{\mathrm{e}}$ and $T_{\mathrm{i}}$ as two independent factors operating within each tidal cycle (emersionimmersion) to co-limit predator performance (prey consumption rate during emersion). We assumed that the responses to $T_{\mathrm{e}}$ and $T_{\mathrm{i}}$ are both unimodal TPC, $f_{\mathrm{e}}\left(T_{\mathrm{e}}\right)$ and $f_{\mathrm{i}}\left(T_{\mathrm{i}}\right)$, respectively, each with a unique set of parameters (Appendix S3). As we are not aware of any published model on how the two factors co-limit the performance of intertidal rates, we assumed the following simple multiplicative model:

$$
p\left(T_{\mathrm{e}}, T_{\mathrm{i}}\right)=p_{\max } f_{\mathrm{e}}\left(T_{\mathrm{e}}\right) f_{\mathrm{i}}\left(T_{\mathrm{i}}\right)
$$

where $p\left(T_{\mathrm{e}}, T_{\mathrm{i}}\right)$ is the per capita predation rate in a tidal cycle and $p_{\max }$ its maximal possible value (constant). The parameters of the model were adjusted empirically to fit the experimental observations under various combinations of constant $T_{\mathrm{e}}$ and $T_{\mathrm{i}}$ conditions (Appendix S3: Fig. $\mathrm{S} 1)$. The generated model can therefore be used as the mean-field in Eq. 1 and, as we show below, it allows formulating mathematically grounded null hypotheses for the effects of the variance in each factor as well as the effects of their covariance and co-limitation.

\section{From coincidence to covariance}

In order to generate the ST predictions, we quantified the means, variances, and covariances of $T_{\mathrm{e}}$ and $T_{\mathrm{i}}$ applied in each of the experimental treatments in Pincebourde et al. (2012). In the original study, the authors did not quantify the covariance in $T_{\mathrm{e}}$ and $T_{\mathrm{i}}$. Instead, they quantified the degree of coincidence between stressful $T_{\mathrm{e}}$ and $T_{\text {i }}$, i.e., the relative proportion of the tidal cycles (during the entire duration of the experiment) at which $T_{\mathrm{e}}$ and $T_{\mathrm{i}}$ above a given threshold coincided. In essence, the degree of "coincidence" in extreme $T_{\mathrm{e}}$ and $T_{\mathrm{i}}$ temperatures depends on the correlation between the fluctuations of the two factors. When scaled to the respective amplitudes of the fluctuations of $T_{\mathrm{e}}$ and $T_{\mathrm{i}}$, this correlation corresponds to their level of covariance. Hence, the extreme high $T_{\mathrm{e}}$ and $T_{\mathrm{i}}$ coincide $(100 \%$ coincidence) when their covariance is maximal (strongly positive). The covariance is minimal (strongly negative) when the $T_{\mathrm{e}}$ and $T_{\mathrm{i}}$ peaks do not coincide $(0 \%$ coincidence). Finally, in the case of partial coincidence $(50 \%)$ treatments the covariance is close to zero (slightly positive or negative).

\section{Exploring the relationship between coincidence and the mean thermal conditions}

In their study, Pincebourde et al. (2012) generated a series of theoretical predictions (Fig. 6) subsequently used as null hypotheses for their experiments. These predictions were generated by averaging the consumption rates they observed under constant temperature conditions. In essence, their predictions correspond to the integrated performance, $\overline{p\left(T_{\mathrm{i}}, T_{\mathrm{e}}\right)}$, introduced in Eq. 1. 


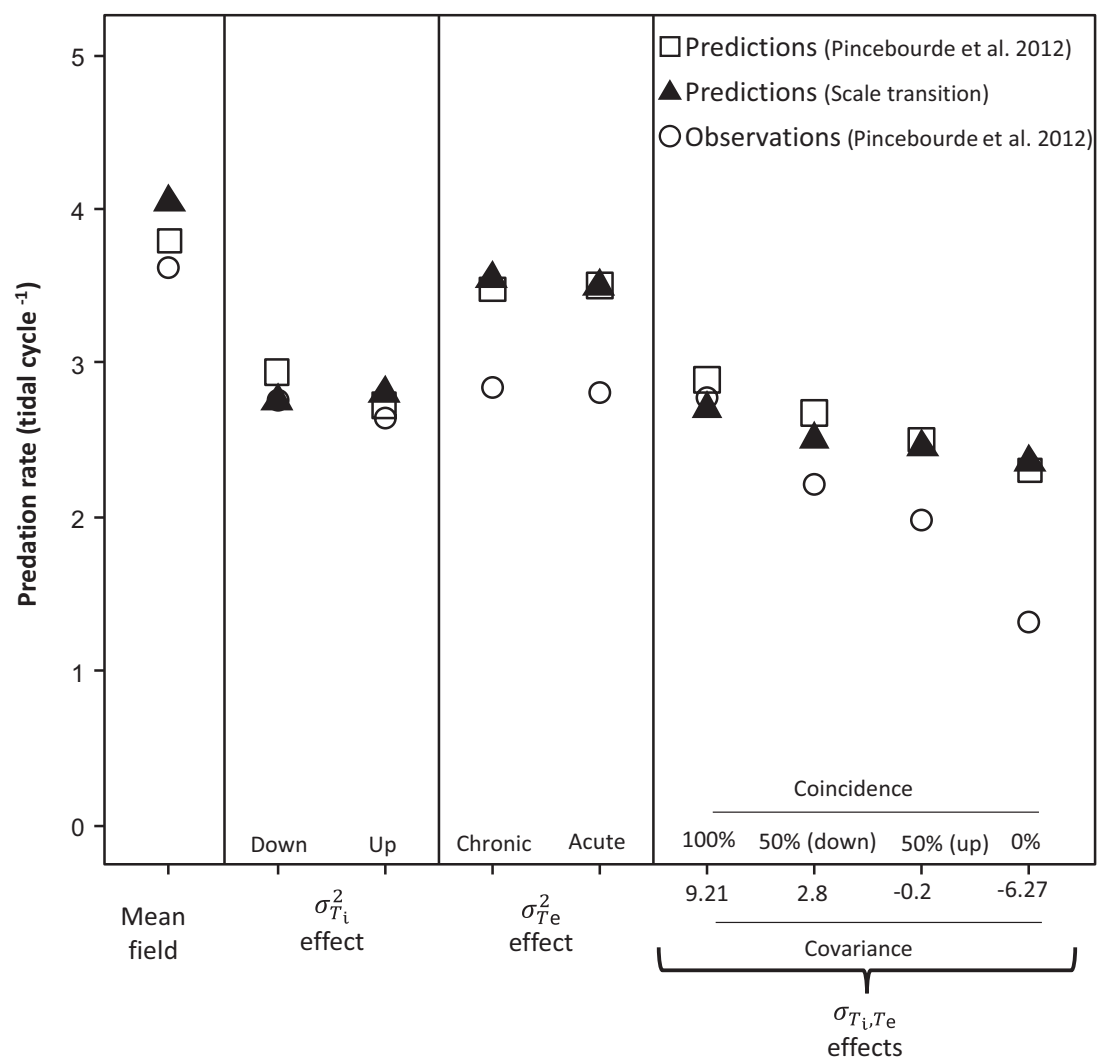

FIG. 6. Revising thermal stress coincidence. Theoretical predictions and experimental observations of the per capita feeding rate of Pisaster ochraceus under the various thermal treatments in Pincebourde et al. (2012). These treatments consisted in manipulating the variance of immersion $\left(T_{\mathrm{i}}\right)$ and emersion $\left(T_{\mathrm{e}}\right)$ body temperatures. We recalculated the covariance values from the data available in Pincebourde et al. (2012). See Appendix S3 for model equations and parameters

Indeed, by using our theoretical model for temperaturedependent predation rate (Eq. 4) with the ST approach we obtained very similar predictions (Fig. 6). Yet, in contrast to the original predictions, ours are mathematically linked to (1) clearly identified features of the temperature reaction norm of the predator (nonlinearities) and (2) to the statistical structure of the immersed and emersed thermal environments experienced by the predator (mean, variance, and covariance).

For example, the prediction of lower average predation rates under variable $T_{\mathrm{i}}$ compared to variable $T_{\mathrm{e}}$ conditions (Fig. 7) despite twofold higher thermal variance applied in the latter $\left(7^{\circ}\right.$ and $15^{\circ} \mathrm{C}^{2}$, respectively) is due to the much higher curvature along the $T_{\mathrm{i}}$ axis (i.e., $\left.p_{T_{\mathrm{i}}, T_{\mathrm{i}}}^{\prime \prime}\left(\overline{T_{\mathrm{i}}}, \overline{T_{\mathrm{e}}}\right)>p_{T_{\mathrm{e}}, T_{\mathrm{e}}}^{\prime \prime}\left(\overline{T_{\mathrm{i}}}, \overline{T_{\mathrm{e}}}\right)\right)$. More interestingly, the apparently counter-intuitive result that the frequent coincidence of stressful $T_{\mathrm{i}}$ and $T_{\mathrm{e}}$ peaks within a tidal cycle is less harmful for performance than their non-coincidence (Fig. 6) can be explained by the synergistic effects (i.e., $p_{T_{\mathrm{i}}, T_{\mathrm{e}}}^{\prime \prime}\left(\overline{T_{\mathrm{i}}}, \overline{T_{\mathrm{e}}}\right)>0$ ) of the two factors on predation rate. A $100 \%$ coincidence of $T_{\mathrm{i}}$ and $T_{\mathrm{e}}$ stressful events means that lower (more optimal) $T_{\mathrm{i}}$ and $T_{\mathrm{e}}$ also always coincide. The predation rates under these coincident optimal conditions are high enough to compensate for the coincident stressful conditions. More importantly, the synergy (or super-additivity) of $T_{\mathrm{i}}$ and $T_{\mathrm{e}}$ implies that the positive effect of coincident optimal $T_{\mathrm{i}}$ and $T_{\mathrm{e}}$ on the predation rate is higher than the sum of the effects of each factor alone (i.e., when optimal $T_{\mathrm{i}}$ and $T_{\mathrm{e}}$ never coincide).

Under the assumed mean-field model, the ST theory can be used to expand the predictions of the net effects of temperature variability (Fig. 5a) and the relative importance of each factor to these effects (Fig. 5b-d) over a wide range of environmental conditions (see also Appendix S3: Fig. S2). For example, all else being equal, an increase in mean $T_{\mathrm{e}}$ from $21.5^{\circ}$ to $24^{\circ} \mathrm{C}$ should have a stronger negative effect on the average predation rate under noncoincidence (or negative covariance) than coincidence (or positive covariance) conditions $(-24 \%$ and $-17 \%$ respectively; Fig. 7a). Regarding the effect partitioning, the relative effect of $T_{\mathrm{e}}$ variance $\left(\frac{1}{2} p_{T_{\mathrm{i}}, T_{\mathrm{i}}}^{\prime \prime}\left(\overline{T_{\mathrm{i}}}, \overline{T_{\mathrm{e}}}\right) \sigma_{T_{\mathrm{e}}}^{2}\right)$ and of covariance $\left(p_{T_{\mathrm{i}}, T_{\mathrm{e}}}^{\prime \prime}\left(\overline{T_{\mathrm{i}}}, \overline{T_{\mathrm{e}}}\right) \sigma_{T_{\mathrm{i}}, T_{\mathrm{e}}}\right)$ should increase (Fig. 7c, d).

Interestingly, all else being equal, colder mean water temperatures $\left(T_{\mathrm{i}}<12^{\circ} \mathrm{C}\right)$, should lead to an inversion of the covariance effects (Fig. 7c) $\left(p_{T_{\mathrm{i}}, T_{\mathrm{e}}}^{\prime \prime}\left(\overline{T_{\mathrm{i}}}, \overline{T_{\mathrm{e}}}\right)<0\right)$. This implies that negative covariance in $T_{\mathrm{i}}$ and $T_{\mathrm{e}}$ contributes positively to performance, while positive covariance has a negative effect (Fig. 7a). In this case, however, the coincidence of stressors should also be redefined as a negative covariance situation in which suboptimally low $T_{\mathrm{i}}$ (cold 

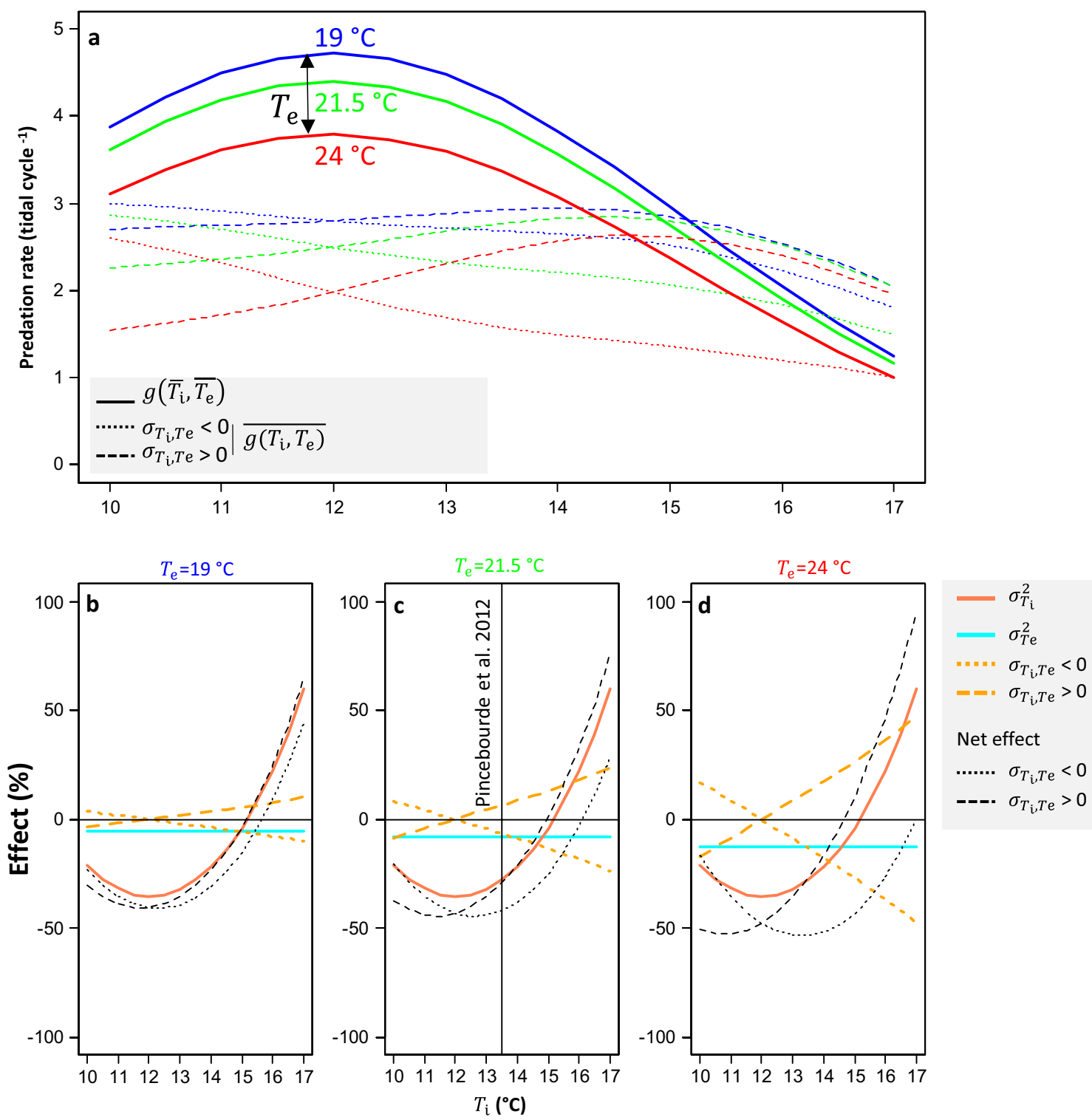

Net effect

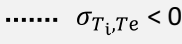

---. $\sigma_{T_{\mathrm{i}}, T e}>0$

FIG. 7. Simulation of immersion $\left(T_{\mathrm{i}}\right)$ and emersion $\left(T_{\mathrm{e}}\right)$ temperature covariance effects on the long-term average per capita predation rate of $P$. ochraceus along a gradient of mean $T_{\mathrm{i}}$ and three mean $T_{\mathrm{e}}$ values. (a) Performance under constant condition (solid lines) vs. performance under variable conditions for maximal (long dashed lines) and minimal covariance (short dashed lines) predicted using the scale transition (ST) approach. (b-d). Effect partitioning using the ST approach. The effects are expressed as deviation (\%) from the mean-field. The net effect is the sum of all the effects under positive or negative covariance conditions. The vertical line depicts the effect partitioning under the mean experimental conditions used in Pincebourde et al. (2012). Simulation parameters $\sigma_{T_{\mathrm{i}}}^{2}=7, \sigma_{T_{\mathrm{e}}}^{2}=15, \sigma_{T_{\mathrm{i}}, T_{\mathrm{e}}}= \pm 10$. See Appendix $\mathrm{S} 3$ for model equations and parameters.

stress) coincides with suboptimally high $T_{\mathrm{e}}$ (heat stress). Consequently, when expressed in terms of stress coincidence, the pattern of the effects should remain unaltered (i.e., a higher performance should be observed under stress coincidence conditions)

\section{Reinterpreting observed deviations}

Under $T_{\mathrm{i}}$ variance alone and under coincident (100\%) fluctuating regime, the observed predator performance reached a level similar to that predicted but was otherwise always below (i.e., for $T_{\mathrm{e}}$ variance alone, $50 \%$ and $0 \%$ coincident fluctuating regimes; Fig. 6). The underlying mechanisms of these deviations being unknown, the authors hypothesized that $P$. ochraceus requires a period free of any thermal stress to either recover from, or compensate for the effects of past exposure. According to this hypothesis, in the $100 \%$ coincidence treatments, episodes of cold water occurring right after periods of concomitant high aerial and underwater thermal stresses could optimize the capacity of $P$. ochraceus to recover or compensate quickly. Here, we use the conceptualization of 
time-dependent effects that we presented above to refine the authors' original hypothesis.

Some observations deviate from our predictions of integrated performance $\overline{p\left(T_{\mathrm{i}}, T_{\mathrm{e}}\right)}$. Referring back to Fig. $4 \mathrm{c}$ and e, this type of deviation is in agreement with our definition of the phenotypic plasticity effect (Box 3; Fig. 4c). When exposed to high temperatures during the low tide, $P$. ochraceus increases feeding rate during the next high tide phase (Pincebourde et al. 2008). This suggests an acclimation aiming to compensate the metabolic costs of high $T_{\mathrm{e}}$ during the following immersion. However, this plastic response seems to be constrained by trade-offs involving a suboptimal performance under low $T_{\mathrm{e}}$ ( $\sigma_{T_{\mathrm{e}}}^{2}$ effect, Fig. 6) and/or low $T_{\mathrm{i}}\left(\sigma_{T_{\mathrm{i}}, T_{\mathrm{e}}}\right.$ effects, $50 \%$ and $0 \%$ coincidence, Fig. 6). This further contributes to the apparently counter-intuitive result of lower performance under lower stress coincidence (effects, 50\% and $0 \%$ coincidence, Fig. 6).

In conclusion, we demonstrated that our framework not only enables a novel theoretical insight into the relationship between thermal stress coincidence and mean thermal conditions in intertidal habitats, but that it also provides a solid re-interpretation of the exact mechanisms contributing to the experimental observations.

\section{Discussion}

\section{Exploring the role of environmental covariance as a driver of physiological performance}

We report that covariance can drive or at least strongly influence integrated physiological performance over a wide range of values of co-limiting factors. The integrated physiological performance becomes sensitive to co-limiting factor covariance when the factors act nonadditively on physiological processes. Hence, the fact that non-additive responses to ecological factor combinations appear to be the rule in nature (Darling and Côté 2008, Jackson et al. 2016) strongly suggests that covariance plays a major yet largely overlooked role in organismal performance (Pincebourde et al. 2012, Koussoroplis and Wacker 2016).

Covariance patterns in nature are highly diverse and depend on the considered factors (Boyd et al. 2014, Gunderson et al. 2016). While only one covariance direction prevails in some combinations (e.g., positive covariance between temperature and drought intensity), the direction and the strength of covariance may vary between habitats, scales, or seasons in other combinations (Gunderson et al. 2016). For example, Reum et al. (2014) showed that the variance in carbonate chemistry, temperature, and oxygen, as well as the strength and the slopes of their correlations in the water column of a fjord, change with season and location, thereby exposing vertical migrators to different covariance patterns. Similarly, depending on the biogeochemical context, phytoplankton density, the main food of freshwater herbivorous zooplankton, may peak in the warmer surface layer or in the deep cold waters thus leading to a positive or negative food-temperature covariance, respectively (Winder et al. 2004). Both situations can occur within the same lake at different times or simultaneously at different locations (Rinke et al. 2009). Despite the examples above and few others, the environmental covariance structures experienced by organisms remain to be determined in most ecological contexts.

Anthropogenic pressure on ecosystems can potentially modify the natural covariance patterns through several ways. Because the covariance in two factors is the product of their SD to their correlation coefficient, a change in the variance of any of the two variables or their spatial or temporal correlation also modifies covariance. For example, there is compelling evidence that thermal variability will change over the next century, both temporally (Easterling et al. 2000, Donat and Alexander 2012) and spatially (Sears et al. 2011, Caillon et al. 2014, Kraemer et al. 2015). All else being equal, these changes should alter the covariance between temperature and any other temporally or spatially correlated co-limiting factor. Yet, covariance might also change independently from variance. In a thermally heterogeneous landscape, an increase in mean temperature may homogenize the spatial distribution of prey or skew it toward the relatively cooler patches, thus driving resource-temperature covariance experienced by ectotherm consumers toward zero or more negative values, respectively (Huey 1991, Davies et al. 2006). Changes in other factors, such as competition or predation, might also drive the prey toward different thermal habitats, thus modifying the food-temperature covariance experienced by their consumers.

Our framework provides two important insights on when and how the covariance structure experienced by organisms and its potential alterations influence the physiological performance. The first is that synergy and antagonism of the co-limiting factors determine whether a given covariance direction (positive or negative) improves or reduces physiological performance. Yet, in some cases, the effect of two co-limiting factors on physiological performance can switch from synergistic to antagonistic or additive depending on the mean values of the factors (Sperfeld et al. 2016) indicating that the direction and the magnitude of covariance effects is context specific but still predictable. The second important insight is that depending on the complexity of the interaction between the co-limiting factors, the sensitivity to co-limiting factor covariance might peak either at the edges (e.g., close to the critical points of the TPC) or close to the center (e.g., close to the thermal optimum of the TPC) of the ecological niche of a species. In the first case, the species is sensitive to stressor coincidence (i.e., extreme co-limiting factor values), even in the absence of physiological damage or compensatory responses (Gunderson et al. 2016, Taff and Vitousek 2016), and covariance patterns potentially determine the spatiotemporal limits of the species distribution. In the second case, covariance will determine the species performance in its 
preferred habitat and covariance patterns potentially set the population growth rate.

\section{Applying the framework to global change biology: challenges and perspectives}

Understanding the mechanistic details of organismal physiological performance increases considerably our ability to predict and anticipate the effects of global change at the scales of biotic communities (Helmuth et al. 2005). In this context, the roles of (1) environmental (to date, mostly thermal) heterogeneity (e.g., Potter et al. 2013, Vasseur et al. 2014, Dowd et al. 2015), (2) the interactions between temperature and other drivers of global change (e.g., Cross et al. 2015, Gunderson et al. 2016), and (3), the phenotypic plasticity of the organisms facing environmental changes (Gunderson et al. 2016, Taff and Vitousek 2016) emerge as major research issues. Here, all these aspects are united into a common conceptual framework applicable for designing experiments, interpreting experimental and field data, and eventually predicting the biological impacts of ongoing global change. While employing the framework for designing and interpreting controlled lab experiments is rather straightforward (Koussoroplis and Wacker 2016), applying it for testing hypotheses or generating predictions in more complex field contexts involves certain challenges, which, once addressed, open new perspectives.

Each organism experiences the environment and its variations at the spatial and temporal scales imposed by body size and its resulting properties (e.g., metabolic rates, mobility, longevity; Woods et al. 2015). Thus, a first challenge is to measure in parallel the environmental factors of interest at the scales that are relevant for the organisms. For example, biomimetic data logging is a promising method for obtaining organism-relevant high resolution (both spatial and temporal) data sets for body temperature (Helmuth et al. 2010). Biophysical modeling can be an important alternative approach to extensive data logging, yet quite demanding in terms of parameter determination (Helmuth et al. 2005). These approaches should be extended to obtain parallel temporal series for factors such as $\mathrm{pH}$, dissolved oxygen, salinity, and other physical constraints. This would enable a baseline understanding of how all these potentially co-limiting factors covary at scales relevant to organisms.

Usually, organisms experience environmental fluctuations (Vasseur and Yodzis 2004, Dillon et al. 2016) with different effects on performance among temporal grains. Therefore, a great challenge underlying the estimation of the biological impacts of climatic change is to measure the amount of variance experienced by organisms at the different temporal scales. A promising tool for achieving this is spectral analysis. It uses Fourier transforms to decompose a time series (e.g., body temperature) into sine waves of different amplitudes (variance or power) and frequencies (Dillon et al. 2016). Used on field time series, this approach enables the quantification of the contribution of each frequency to the total variation. This can be very useful for identifying the temporal scales at which environmental variation most strongly influences performance and for designing experiments addressing the impacts of multiscale environmental variation (Dillon et al. 2016). There is a promising opportunity to operate a fruitful connection between ST theory and spectral analysis (Denny 2016), which can be readily extended to embrace the multifactorial perspective of our framework (e.g., by using metrics such as cross-covariance or coherence) and to assess how co-limiting factors covary at different temporal scales.

In nature, the physiological responses to environmental variation cannot be decoupled from behavior as these two aspects interact. A moving individual samples space at a given rate, causing a unique value of variance that integrates both space (e.g., spatial heterogeneity of temperatures) and time (e.g., sampling rate of patches and temporal variability within the patches; Woods et al. 2015). Behavior is permanently adjusted according to environmental conditions and to the status of the organism (e.g., its thermal needs or nutritional state). For example, many organisms exhibit thermoregulatory behaviors and/or will track to a certain extent resource peaks in the landscape thereby acting as a strong filter of environmental temperature variance (Sears and Angilletta 2015; Pincebourde et al. 2016). Hence, fine tracking of organisms' movements in their habitat is essential for understanding how they integrate and filter the heterogeneity of their environment into parallel time-series of co-limiting factors. In that aim, recent technological progress offers a large panel of bio-logging and bio-tracking methods, applicable even to small organisms such as insects (Le Galliard et al. 2012).

Sclerochronology offers a highly promising alternative or complementary approach, yet limited to organisms with incremental sclerified structures. For example, by combining microchemical and microscopic methods, otoliths can provide parallel time series of proxies for several potentially co-limiting factors including temperature, food intake, and water chemistry (Morrongiello et al. 2012). These time series are individual, integrate both environmental variability and behavior, and in some cases, can be highly resolved (up to the daily scale; Morrongiello et al. 2012). Furthermore, otoliths offer the possibility to estimate performance (growth rate), both at short and long time scales. Combined with pre-established performance curves, such otolith-derived data sets could offer a formidable opportunity to test and apply our framework and contribute powerful insights on how ongoing multifactorial changes in the statistical structure of the environment affect individual performance.

Pre-established performance curves and surfaces are essential for the application of the framework. Performance curves for a large diversity of factors (temperature, food, light, etc.) and organisms are readily available in the literature, yet performance surfaces are still relatively rare. This is partially due to the relatively recent inclusion of multiple factors in experimental global change biology but 
mostly to the predominance of factorial ANOVA-oriented designs incorporating only few highly replicated factor levels (usually two to three). Our framework underscores the insights to be gained by shifting from factorial to gradient designs (sensu Sperfeld et al. 2016) in experimental global change biology. Gradient experimental designs, consist in measuring performance over a continuous multifactorial state space. The effort of increasing the range of tested values very likely imposes a decrease in treatment replication. We argue that this cost is worthwhile because the question is no longer to verify whether there is a significant interaction between two environmental factors but to understand how this interaction changes in the variable state space.

An interesting alternative to experiments is physiological modeling, but it also involves experimental determination of several parameters. Physiological modeling, potentially combined with experiments, offers a promising avenue for a tighter theoretical integration of timedependent effects in our framework (e.g., Kingsolver and Woods 2016). Although further theoretical developments are required, the models could be used to generate response surfaces with temporal scale (fluctuation frequency) as a factor co-limiting performance (Rezende et al. 2014) and thus to explicitly incorporate time scale as a factor in the ST equations. Such an integration would allow one to address aspects such as periodicity (time scale has no variance) or stochastity (time scale has a variance) of environmental fluctuations. For all their detail, physiological models do not question the necessity of the ST approach. Indeed, slightly paraphrasing Chesson (2012): ST is a biological theory. It is not intended to be a better way of solving models or performing calculations. ST is about quantitative concepts (curvature, variance, and covariance) that explain the behavior of biological systems. Finally, there is much to be gained by playing with the high flexibility of the ST theory in a multifactorial context.

\section{ACKNOWLEDGMents}

We thank Mark Denny, two anonymous reviewers, and the editor (Brian Inouye) for valuable comments on earlier versions of this manuscript. This study was supported by the German Research Foundation (DFG: WA2445/8-1, WA2445/9-1 and KO5330/1-1) and by the Agence National pour la Recherche (MicroCliMite, ANR-2010 BLAN-1706-02 to S. Pincebourde).

\section{Literature Cited}

Angilletta, M. J. 2006. Estimating and comparing thermal performance curves. Journal of Thermal Biology 31:541-545.

Arendt, J. D. 1997. Adaptive intrinsic growth rates: an integration across taxa. Quarterly Review of Biology 72:149-177.

Bauerfeind, S. S., and K. Fischer. 2013. Increased temperature reduces herbivore host-plant quality. Global Change Biology 19:3272-3282.

Benedetti-Cecchi, L., I. Bertocci, S. Vaselli, and E. Maggi. 2006. Temporal variance reverses the impact of high mean intensity of stress in climate experiments. Ecology 87: 2489-2499.
Blanford, J. I., S. Blanford, R. G. Crane, M. E. Mann, K. P. Paaijmans, K. V. Schreiber, and M. B. Thomas. 2013. Implications of temperature variation for malaria parasite development across Africa. Scientific Reports 3:1300.

Boyd, P. W., S. T. Lennartz, D. M. Glover, and S. C. Doney. 2014. Biological ramifications of climate-change-mediated oceanic multi-stressors. Nature Climate Change 5:71-79.

Caillon, R., C. Suppo, J. Casas, H. Arthur Woods, and S. Pincebourde. 2014. Warming decreases thermal heterogeneity of leaf surfaces: implications for behavioural thermoregulation by arthropods. Functional Ecology 28:1449-1458.

Chesson, P. 2012. Scale transition theory: its aims, motivations and predictions. Ecological Complexity 10:52-68.

Chesson, P., M. J. Donahue, B. Melbourne, and A. L. Sears. 2005. Scale transition theory for understanding mechanisms in metacommunities. Pages 279-306 in M. Holoyak, M. A. Leibold, and R. D. Holt, editors. Metacommunities: dynamics and ecological communities. Wiley, Chicago, Illinois, USA.

Cross, W. F., J. M. Hood, J. P. Benstead, A. D. Huryn, and D. Nelson. 2015. Interactions between temperature and nutrients across levels of ecological organization. Global Change Biology 21:1025-1040.

Darling, E. S., and I. M. Côté. 2008. Quantifying the evidence for ecological synergies. Ecology Letters 11:1278-1286.

Davies, Z. G., R. J. Wilson, S. Coles, and C. D. Thomas. 2006. Changing habitat associations of a thermally constrained species, the silver-spotted skipper butterfly, in response to climate warming. Journal of Animal Ecology 75:247-256.

De Senerpont Domis, L. N., D. B. Van de Waal, N. R. Helmsing, E. Van Donk, and W. M. Mooij. 2014. Community stoichiometry in a changing world: combined effects of warming and eutrophication on phytoplankton dynamics. Ecology 95: 1485-1495.

Denny, M. W. 2016. Ecological mechanics principles of life's physical interactions. Princeton University Press, Princeton, New Jersey, USA.

Denny, M., and L. Benedetti-Cecchi. 2012. Scaling up in ecology: mechanistic approaches. Annual Review of Ecology Evolution and Systematics 43:1-22.

Dillon, M. E., H. A. Woods, G. Wang, S. B. Fey, D. A. Vasseur, R. S. Telemeco, et al. 2016. Life in the frequency domain: the biological impacts of changes in climate variability at multiple time scales. Integrative and Comparative Biology 56: $14-30$.

Donat, M. G., and L. V. Alexander. 2012. The shifting probability distribution of global daytime and night-time temperatures. Geophysical Research Letters 39:L14707, doi:10.1029/2012GL052459.

Dowd, W. W., F. A. King, and M. W. Denny. 2015. Thermal variation, thermal extremes and the physiological performance of individuals. Journal of Experimental Biology 218: 1956-1967.

Easterling, D. R., G. A. Meehl, C. Parmesan, S. A. Changnon, T. R. Karl, and L. O. Mearns. 2000. Climate extremes: observations, modeling, and impacts. Science 289:2068-2074.

Edwards, K. F., M. K. Thomas, C. A. Klausmeier, and E. Litchman. 2016. Phytoplankton growth and the interaction of light and temperature: a synthesis at the species and community level: light-temperature interactions. Limnology and Oceanography 61:1232-1244.

Estay, S. A., M. Lima, and F. Bozinovic. 2014. The role of temperature variability on insect performance and population dynamics in a warming world. Oikos 123:131-140.

Fox, J. 2016. A painless introduction to partitions in ecology and evolution. Web blog post. Dynamic Ecology, 6 June 2016. https://dynamicecology.wordpress.com 
Fujiwara, M., B. E. Kendall, and R. M. Nisbet. 2003. Growth autocorrelation and animal size variation: growth autocorrelation and size variability. Ecology Letters 7: 106-113.

Gunderson, A. R., E. J. Armstrong, and J. H. Stillman. 2016. Multiple stressors in a changing world: the need for an improved perspective on physiological responses to the dynamic marine environment. Annual Review of Marine Science 8:357-378.

Gurney, W. S. C., W. Jones, A. R. Veitch, and R. M. Nisbet. 2003. Resource allocation, hyperphagia, and compensatory growth in juveniles. Ecology 84:2777-2787.

Harpole, W. S., J. T. Ngai, E. E. Cleland, E. W. Seabloom, E. T. Borer, M. E. S. Bracken, et al. 2011. Nutrient co-limitation of primary producer communities: community co-limitation. Ecology Letters 14:852-862.

Helmuth, B., et al. 2010. Organismal climatology: analyzing environmental variability at scales relevant to physiological stress. Journal of Experimental Biology 213:995-1003.

Helmuth, B., J. G. Kingsolver, and E. Carrington. 2005. Biophysics, physiological ecology, and climate change: does mechanism matter? Annual Review of Physiology 67: 177-201.

Hood, J., and R. Sterner. 2010. Diet mixing: Do animals integrate growth or resources across temporal heterogeneity? American Naturalist 167:651-663.

Huey, R. B. 1991. Physiological consequences of habitat selection. American Naturalist 137:91-115.

Jackson, M. C., C. J. G. Loewen, R. D. Vinebrooke, and C. T. Chimimba. 2016. Net effects of multiple stressors in freshwater ecosystems: a meta-analysis. Global Change Biology 22: $180-189$.

Jensen, J. L. W. V. 1906. Sur les fonctions convexes et les inégalités entre les valeurs moyennes. Acta Mathematica 30: 175-193.

Kingsolver, J. G., J. K. Higgins, and K. E. Augustine. 2015. Fluctuating temperatures and ectotherm growth: distinguishing non-linear and time-dependent effects. Journal of Experimental Biology 218:2218-2225.

Kingsolver, J. G., and H. A. Woods. 2016. Beyond thermal performance curves: modeling time-dependent effects of thermal stress on ectotherm growth rates. The American Naturalist 187:283-294.

Kooijman, B. 2010. Dynamic energy budget theory for metabolic organisation. Third edition. University Press, Cambridge, UK.

Koussoroplis, A.-M., and A. Wacker. 2016. Covariance modulates the effect of joint temperature and food variance on ectotherm life-history traits. Ecology Letters 19:143-152.

Kraemer, B. M., O. Anneville, S. Chandra, M. Dix, E. Kuusisto, D. M. Livingstone, et al. 2015. Morphometry and average temperature affect lake stratification responses to climate change: lake stratification responses to climate. Geophysical Research Letters 42:4981-4988.

Le Galliard, J.-F., J.-M. Guarini, F. Gaill, and Institut Écologie et environnement (France). 2012. Sensors for ecology: towards integrated knowledge of ecosystems. CNRS, Institut écologie et environnement, Paris, France

Lemoine, N., and D. Burkepile. 2012. Temperature-induced mismatches between consumption and metabolism reduce consumer fitness. Ecology 93:2483-2489.

Litchman, E. 2000. Growth of phytoplankton under fluctuating light. Freshwater Biology 44:223-235.

Malzahn, A. M., D. Doerfler, and M. Boersma. 2016. Junk food gets healthier when it's warm: temperature and food quality interactions on A. Tonsa. Limnology and Oceanography 61:1677-1685.
Martin, T. L., and R. B. Huey. 2008. Why "suboptimal" is optimal: Jensen's inequality and ectotherm thermal preferences. American Naturalist 171:E102-E118.

Matassa, C. M., and G. C. Trussell. 2014. Prey state shapes the effects of temporal variation in predation risk. Proceedings of the Royal Society B 281:20141952.

Melbourne, B. A., and P. Chesson. 2006. The scale transition: scaling up population dynamics with field data. Ecology 87:1478-1488.

Metcalfe, N. B., and P. Monaghan. 2001. Compensation for a bad start: grow now, pay later? Trends in Ecology \& Evolution 16:254-260.

Morozov, A., and J.-C. Poggiale. 2012. From spatially explicit ecological models to mean-field dynamics: the state of the art and perspectives. Ecological Complexity 10:1-11.

Morrongiello, J. R., R. E. Thresher, and D. C. Smith. 2012. Aquatic biochronologies and climate change. Nature Climate Change 2:849-857.

Muller, E. B., and R. M. Nisbet. 2000. Survival and production in variable resource environments. Bulletin of Mathematical Biology 62:1163-1189.

Niehaus, A. C., M. J. Angilletta, M. W. Sears, C. E. Franklin, and R. S. Wilson. 2012. Predicting the physiological performance of ectotherms in fluctuating thermal environments. Journal of Experimental Biology 215:694-701.

Orians, C. M., and C. G. Jones. 2001. Plants as resource mosaics: a functional model for predicting patterns of within-plant resource heterogeneity to consumers based on vascular architecture and local environmental variability. Oikos 94:493-504.

Paaijmans, K. P., S. Blanford, A. S. Bell, J. I. Blanford, A. F. Read, and M. B. Thomas. 2010. Influence of climate on malaria transmission depends on daily temperature variation. Proceedings of the National Academy of Sciences USA 107:15135-15139.

Park, S., S. Chandra, D. C. Müller-Navarra, and C. R. Goldman. 2004. Diel and vertical variability of seston food quality and quantity in a small subalpine oligomesotrophic lake. Journal of Plankton Research 26:1489-1498.

Pincebourde, S., C. C. Murdock, M. Vickers, and M. W. Sears. 2016. Fine-scale microclimatic variation can shape the responses of organisms to global change in both natural and urban environments. Integrative and Comparative Biology $56: 45-61$.

Pincebourde, S., E. Sanford, J. Casas, and B. Helmuth. 2012. Temporal coincidence of environmental stress events modulates predation rates: predation in fluctuating environments. Ecology Letters 15:680-688.

Pincebourde, S., E. Sanford, and B. Helmuth. 2008. Body temperature during low tide alters the feeding performance of a top intertidal predator. Limnology and Oceanography 53:1562-1573.

Potter, K. A., H. Arthur Woods, and S. Pincebourde. 2013. Microclimatic challenges in global change biology. Global Change Biology 19:2932-2939.

Retkute, R., S. E. Smith-Unna, R. W. Smith, A. J. Burgess, O. E. Jensen, G. N. Johnson, et al. 2015. Exploiting heterogeneous environments: does photosynthetic acclimation optimize carbon gain in fluctuating light? Journal of Experimental Botany 66:2437-2447.

Reum, J. C. P., S. R. Alin, R. A. Feely, J. Newton, M. Warner, and P. McElhany. 2014. Seasonal carbonate chemistry covariation with temperature, oxygen, and salinity in a fjord estuary: implications for the design of ocean acidification experiments. PLoS ONE 9:e89619.

Rezende, E. L., L. E. Castañeda, and M. Santos. 2014. Tolerance landscapes in thermal ecology. Functional Ecology 28: 799-809. 
Rhee, G. Y., and I. Gotham. 1981. The effect of environmental factors on phytoplankton growth: temperature and the interactions of temperature with nutrient limitation. Limnology and Oceanography 26:638-648.

Rinke, K., A. M. R. Huber, S. Kempke, M. Eder, T. Wolf, W. N. Probst, et al. 2009. Lake-wide distributions of temperature, phytoplankton, zooplankton, and fish in the pelagic zone of a large lake. Limnology and Oceanography 54:1306-1322.

Ruel, J. J., and M. P. Ayres. 1999. Jensen's inequality predicts effects of environmental variation. Trends in Ecology \& Evolution 14:361-366.

Schulte, P. M., T. M. Healy, and N. A. Fangue. 2011. Thermal performance curves, phenotypic plasticity, and the time scales of temperature exposure. Integrative and Comparative Biology 51:691-702.

Sears, M. W., E. Raskin, and M. J. Angilletta. 2011. The world is not flat: defining relevant thermal landscapes in the context of climate change. Integrative and Comparative Biology 51:666-675.

Sears, M. W., and M. J. Angilletta. 2015. Costs and benefits of thermoregulation revisited: both the heterogeneity and spatial structure of temperature drive energetic costs. The American Naturalist 185:E94-E102.

Simpson, S. J., and D. Raubenheimer. 2012. The nature of nutrition a unifying framework from animal adaptation to human obesity. Princeton University Press, Princeton, New Jersey, USA.

Sinclair, B. J., et al. 2016. Can we predict ectotherm responses to climate change using thermal performance curves and body temperatures? Ecology Letters 19:1372-1385.

Smallwood, P. D. 1996. An introduction to risk sensitivity: the use of Jensen's inequality to clarify evolutionary arguments of adaptation and constraint. American Zoologist 36: 392-401.

Sperfeld, E., D. Martin-Creuzburg, and A. Wacker. 2012. Multiple resource limitation theory applied to herbivorous consumers: Liebig's minimum rule vs. interactive colimitation. Ecology Letters 15:142-150.

Sperfeld, E., D. Raubenheimer, and A. Wacker. 2016. Bridging factorial and gradient concepts of resource co-limitation: towards a general framework applied to consumers. Ecology Letters 19:201-215.
Sperfeld, E., and A. Wacker. 2012. Temperature affects the limitation of Daphnia magna by eicosapentaenoic acid, and the fatty acid composition of body tissue and eggs. Freshwater Biology 57:497-508.

Sterner, R., and M. Schwalbach. 2001. Diel integration of food quality by Daphnia: luxury consumption by a freshwater planktonic herbivore. Limnology and Oceanography 42:410-416.

Stevenson, R. 1985. Body size and limits to the daily range of body temperatures in terrestrial ectotherms. American Naturalist 125:102-117.

Taff, C. C., and M. N. Vitousek. 2016. Endocrine flexibility: optimizing phenotypes in a dynamic world? Trends in Ecology \& Evolution 31:476-488.

Terblanche, J. S., A. A. Hoffmann, K. A. Mitchell, L. Rako, P. C. le Roux, and S. L. Chown. 2011. Ecologically relevant measures of tolerance to potentially lethal temperatures. Journal of Experimental Biology 214:3713-3725.

Tilman, D. 1982. Resource competition and community structure. Princeton University Press, Chichester, West Sussex, UK.

Tremmel, M., and C. Müller. 2013. The consequences of alternating diet on performance and food preferences of a specialist leaf beetle. Journal of Insect Physiology 59:840-847.

Underwood, N. 2004. Variance and skew of the distribution of plant quality influence herbivore population dynamics. Ecology 85:686-693.

Vasseur, D. A., J. P. DeLong, B. Gilbert, H. S. Greig, C. D. G. Harley, K. S. McCann, et al. 2014. Increased temperature variation poses a greater risk to species than climate warming. Proceedings of the Royal Society B 281:20132612.

Vasseur, D. A., and P. Yodzis. 2004. The color of environmental noise. Ecology 85:1146-1152.

Wetzel, W. C., H. M. Kharouba, M. Robinson, M. Holyoak, and R. Karban. 2016. Variability in plant nutrients reduces insect herbivore performance. Nature. https://doi. org/10.1038/nature20140(2016)

Winder, M., P. Spaak, and W. M. Mooij. 2004. Trade-offs in daphnia habitat selection. Ecology 85:2027-2036.

Woods, H. A., M. E. Dillon, and S. Pincebourde. 2015. The roles of microclimatic diversity and of behavior in mediating the responses of ectotherms to climate change. Journal of Thermal Biology 54:86-97.

\section{SUPPORTING INFORMATION}

Additional supporting information may be found in the online version of this article at http://onlinelibrary.wiley.com/ doi/10.1002/ecm.1247/suppinfo 